\title{
Genome wide identification and characterization of cucumber bHLH family genes and the functional characterization of CsbHLH041 in $\mathrm{NaCl}$ and ABA tolerance in Arabidopsis and cucumber
}

Jialin Li

Shandong Agricultural University

Ting Wang

Shandong Agricultural University

Jing Han

Shandong Agricultural University

Zhonghai Ren ( $\nabla$ zhren@sdau.edu.cn )

Shandong Agricultural University https://orcid.org/0000-0002-0759-3668

Research article

Keywords: Abiotic stresses, bHLH family, Cucumber, CsbHLH041, Expression patterns, Regulation networks

Posted Date: November 21st, 2019

DOI: https://doi.org/10.21203/rs.2.17576/v1

License: (c) (1) This work is licensed under a Creative Commons Attribution 4.0 International License.

Read Full License

Version of Record: A version of this preprint was published at BMC Plant Biology on June 11th, 2020. See the published version at https://doi.org/10.1186/s12870-020-02440-1. 


\section{Abstract}

Background The basic/helix-loop-helix (bHLH) transcription factor family exists in all three eukaryotic kingdoms as important regulatory components in biological growth and development. To date, there has been relatively little effort to systematically carry out comprehensive genomic and functional analyses of bHLH genes in cucumber ( Cucumis sativus L.).

Results Here, a total of $142 \mathrm{bHLH}$ genes were identified in the cucumber genome released recently and further classified into 32 subgroups based on the phylogenetic analysis, conserved motifs and gene structures. Multiple sequence alignment analyses showed that the sequences of CsbHLH proteins were highly conserved. The chromosomal distribution, synteny analysis, gene duplications of these 142 CsbHLHs were further analyzed. A cis-element analysis revealed that there were many elements related to stress responsiveness and plant hormones in the promoter regions of CsbHLH genes. Phylogenetic comparison of the bHLH members between cucumber and Arabidopsis, revealed cucumber bHLH proteins were clustered into the different functional clades of Arabidopsis bHLH members. The transcript abundance analysis of selected $\mathrm{CsbHLH}$ under abiotic stresses ( $\mathrm{NaCl}, \mathrm{ABA}$ and low temperature treatments) identified five CsbHLH genes could simultaneously respond to the three abiotic stresses. Tissue-specific expression profiles of these five genes were also analyzed. In addition, 35S:CsbHLH041 enhanced the tolerance to salt and $A B A$ in transgenic Arabidopsis and in cucumber seedlings, suggesting $\mathrm{CsbHLH041}$ is an important regulator in response to abiotic stresses. Finally, the functional interoperability network among the CsbHLH proteins was analyzed.

Conclusion This study provided a good foundation for further research into the functions and regulatory mechanisms of CsbHLH proteins and identified candidate genes for stress resistance in cucumber.

\section{Background}

Basic helix-loop-helix (bHLH) proteins exist widely in all three eukaryotic kingdoms and form one of the largest families of TFs [1, 2]. The bHLH TFs are named for its own structural characteristics [3], which are composed of approximately conservative 60 amino acid residues. According to the different functions, it can be divided into two parts: basic region and HLH [4]. The basic region is distributed at the N-terminal of the bHLH conservative domain and contains approximately 15 to 20 residues, which is involved in DNA binding. Certain conserved amino acids in the basic region determine the recognition of the cisacting element E-box ( 5 '-CANNTG-3'), while other residues will provide specificity for specific types of Ebox. In addition, flanking nucleotides outside of the hexanucleotide core have been shown to play a role in binding specificity $[5,6]$. The HLH domain is distributed at the C-terminal of the gene sequence, which is composed of two amphipathic a-helices mainly consisting of hydrophobic residues linked by a loop region of variable sequence and length. The HLH domain is an essential structure for the formation of homologous or heterologous dimers in bHLH transcription factors [6, 7]. 
According to evolutionary origin, sequence similarity, DNA binding patterns, and functional types, in animals, bHLH transcription factors are mainly divided into A-F Six categories, containing 45 subgroups $[8,9]$. In plants, the $b H L H$ gene family has been divided into 15-26 groups [10], and even up to 32 when atypical bHLH proteins are included [2]. In Arabidopsis, $167 \mathrm{bHLH}$ proteins are divided into 21 subfamilies [2,11]; The $165 \mathrm{bHLH}$ family members in rice are classified into 22 subfamilies [12]; In tomato, $159 \mathrm{bHLH}$ proteins are divided into 21 subfamilies [13]. Nowadays, more and more bHLH proteins are found in plants, and their functional research is gradually deepening.

In plants, the $b H L H$ gene family plays important roles in plant growth and development, metabolic regulation, and response to environmental changes. The first member of the $b H L H$ family discovered was the maize $R$ gene, which was shown to play a key role in anthocyanin synthesis [14]. Subsequently, more and more bHLHs have been shown to be involved in a wider range of physiological pathways. For example, Phytochrome Interacting Factors (PIFs) have been reported to response to light signals [15]; Overexpression of PRE1 activates gibberellin-dependent responses in Arabidopsis thaliana [16]; AtGL3, AtEGL3 and AtTT8 have been demonstrated to be involved in anthocyanin and PA biosynthesis [17, 18], while AtGL3, AtEGL3 and AtMYC1 were also regulate trichome formation and root hair patterning [19]. In addition, some bHLH TFs have also been suggested to respond to diverse abiotic stresses and improve plant stress tolerance, including to cold, salt and drought. In wheat, bHLH39 increases the expression levels of stress-response genes under salt stress, thus improving the salt tolerance of overexpressing bHLH39 wheat plants [20]. VabHLH1 and VvbHLH1 act as positive regulators of the cold stress response, modulating the level of COR gene expression, which in turn confer tolerance to cold stress [21]. The bHLH TFs often function by forming homologous or heterodimer with other proteins. Studies have shown that ZmZOU and ZmICEa form a complex through heterogenous dimerization and have functional effects only in the development of monocotyledonous seeds [22]. So far, the most studied are MYB-bHLH, JAZbHLH, MYB-bHLH-WD40 interacting proteins. For example, MYC3 and MYC4 transcription factors all can interact with multiple JAZ proteins (such as JAZ1, JAZ4, JAZ9) to jointly regulate the JA signal pathway [23]. The MYB-bHLH-WD40 complexs are involved in different processes such as biosynthesis of anthocyanins and PAs, leaf trichome formation and root hair patterning [24]. To sum up, bHLH in plants can form homologous or heterologous complexes with bHLH proteins or other proteins to extend their biological functions.

Cucumber (Cucumis sativus L.) is an economically important crop cultivated worldwide [25]. Although so many important functions of the AtbHLH family in Arabidopsis thaliana have been widely studied [2], genome-wide information of $C s b H L H$ family members has not been reported previously. In this study, we identified and characterized $142 \mathrm{bHLH}$ family genes in cucumber. They were distributed over seven chromosomes and could be classified into 32 subgroups based on phylogenetic analysis. We also investigated their gene structures, conserved motifs, synteny analysis, gene duplications and cis-elements in promoters. In addition, the expression levels of some CsbHLH genes were measured by qRT-PCR to study their responses to $4^{\circ} \mathrm{C}$ low temperature, salt $(\mathrm{NaCl})$ and $\mathrm{ABA}$ stress, which all tested genes were stress responsive. The protein interaction network among the $\mathrm{CsbHLH}$ proteins was predicted, which could help us understand the possible functional mechanism of CsbHLH proteins. Furthermore, 
Agrobacteriu-mediated transient transformation of cucumber cotyledons and stable transgenic Arabidopsis with overexpression of $\mathrm{CsbHLHO41}$ were increased salt resistance and ABA resistance compared with controls. We hoped that this work would provide a useful resource for subsequent research on the functions and regulatory mechanisms of potentially important CsbHLH proteins that were crucial in modulating abiotic stress responses in cucumber.

\section{Results}

\section{Identification and analysis of cucumber $b H L H$ genes}

To identify $b H L H$ family genes in the cucumber genome, we performed the BlastP program to search against the cucumber genome database by using 166 Arabidopsis bHLH proteins $[2,10]$ and the consensus protein sequences of the bHLH domain, Hidden Markov Model (HMM) profile (PF00010) as queries. We obtained 164 putative members of the $C s b H L H$ family. To confirm reliability $b H L H$ genes in the cucumber genome, we used Pfam (http://pfam.janelia.org/) and SMART (http://smart.emblheidelberg.de/) [26] to search for the presence of the bHLH domain in the amino acid sequences of all 164 proteins. We found that only 142 proteins had the corresponding conservative bHLH domain, which were named $C s b H L H 1$ to $C s b H L H 142$ based on their phylogenies and sequence similarity corresponding to individual AtbHLH proteins. Finally, the specific information on the 142 typical bHLH genes, including gene ID, amino acids length, gene length and chromosomal locations were listed in Table 1 . The lengths of the CsbHLH protein sequences ranged from 84 residues (CsaV3_1G005290) to 960 residues (CsaV3_1G043790),, and the isoelectric points (pl) ranged from 4.57 (CsaV3_2G030090) to 11.79 (CsaV3_6G028530).

\section{Phylogenetic analysis, conserved motif and gene structure analysis of CsbHLH gene family}

To confirm the structural characteristics of CsbHLHs, multiple sequence alignment (MSA) analysis was performed with the $142 \mathrm{CsbHLH}$ proteins. As shown in Fig. 1, one basic region, one loop region and two helix regions were detected in all $142 \mathrm{CsbHLH}$ proteins. And conserved amino acids in bHLH domains, with sequence identity more than $50 \%$, were showed in purple or light blue color (Fig. 1a). Sequence logos were produced using the $142 \mathrm{CsbHLH}$ homologous domain amino acid sequences (Fig. 1b). The CsbHLH proteins in cucumber contain 17 conserved amino acids in the bHLH domain, which were present in the bHLH gene familily of Arabidopsis and Moso bamboo [2,27]. As shown in the Fig. 1b, we could clearly observe that Arg-10, Arg-11, Leu-21 and Leu-53 showed highly conservative (92\%, 87\%, 96\%, and 90\%, respectively) among the $142 \mathrm{CsbHLH}$ proteins. These key amino acid residues play an important role in binding transcription factors to DNA and forming homologous or heterodimers between bHLH proteins or with other TFs $[2,10]$. To better understand the evolutionary relationships within the CsbHLH gene family, an un-rooted neighbor-joining $(\mathrm{NJ})$ phylogenetic tree was constructed based on the alignment of the 
corresponding $142 \mathrm{bHLH}$ complete protein sequences, and they were subdivided into 32 subgroups, designated $\mathrm{C} 1-\mathrm{C} 32$, according to clades with at least 50 \% bootstrap support (Fig. 2a).

To support the phylogenetic analysis, gene structure analysis of $b H L H$ family members was performed. The results showed $\mathrm{Cs} b H \mathrm{LH}$ in the same subgroups presented similar numbers of exons and introns, and without considering the size of the introns, the $C s b H L H$ genes within the same subgroups had similar intron-exon gene structures (Fig. 2c). All members of subgroups 8, 9, 15, 22 and 28 contained the same number of exons and introns, respectively, while the most members in subgroups 5, 12, 20, 23 and 30 shared the same number of exons and introns, respectively. Nevertheless, the number of exons and introns varied greatly among other subgroups (Fig. 2c).

To further investigate the specific motifs of CsbHLH proteins within the same subgroup, 10 distinct conserved motifs were identified by the MEME motif search tool (Fig. 2b). All predicted motifs were identified only once in each CsbHLH protein sequences. $142 \mathrm{CsbHLH}$ proteins contained different numbers of conserved motifs, ranging from two to seven, and motifs 1 and 2 representing one part of the bHLH domain were present in almost CsbHLH proteins (Fig. 2b). Meanwhile, similar motif composition existed in CsbHLH proteins of the same subgroup. For example, members of subgroup 23 all contained motifs 1, 2, 4 and 6, while motif 5 was identified in most of the CsbHLH proteins. Interestingly, some of the specific motifs were absent in certain subgroups. For example, motif 4 was absent in all the members of 1,2 and 3 subgroups (Fig. $2 b$ ).

In general, the results of conserved motif and gene structure analyses further proof the results of phylogenetic analysis, illustrating that proteins within the same subgroup may have similar functions.

\section{Synteny analysis of $b H L H$ genes in cucumber, Arabidpsis and tomato}

To determine the genomic distribution and duplication of $C s b H L H$ genes, firstly, the DNA sequence of each gene was used to search the cucumber genome database using BLASTN. All $142 \mathrm{CsbHLH}$ genes (except CsaV3_UNG229040) could be mapped onto chromosomes 1-7 (Fig. 3a; Table 1; Fig. S2). Although each of the seven cucumber chromosomes contained some CsbHLH genes, the distribution seemed to be uneven (Fig. 3a; Fig. S2). The largest number of $\mathrm{CsbHLH}$ genes was found on chromosome 3 (27 genes), followed by chromosomes 1 ( 26 genes) and 6 ( 26 genes), as well as 21 genes on chromosome 2. Twelve, fifteen and fourteen CsbHLH genes were identified on chromosomes 4, 5 and 7, respectively. Then, according to the description reported by [28] to determine the duplication of $\mathrm{Cs} b \mathrm{HLH}$ genes, the syntenic regions were analyzed by MCscanX software. As shown in Table S1, total 1468 tandem duplication gene pairs and 231 segmental duplication blocks were identified in cucumber genome, respectively. Five tandem duplication gene pairs were obtained in $\mathrm{CsbHLH}$ family (CsbHLHO19 and CsbHLH020; CsbHLH019 and CsbHLH025; CsbHLH120 and CsbHLH125; CsbHLH125and CsbHLH126; CsbHLH038 and CsbHLH101) (Fig. 3a; Table S1). In addition, segmental duplication events 
with seven bHLH gene pairs (CsbHLH112 and CsbHLH127; CsbHLH04O and CsbHLH037; CsbHLH054 and CsbHLH085; CsbHLHO6O and CsbHLH074; CsbHLHOO1 and CsbHLH135; CsbHLH141 and CsbHLH046; CsbHLHO5O and CsbHLH044) were also identified in cucumber (Fig. 3a; Table S1).

In order to further illuminate the phylogenetic mechanisms of cucumber $b H L H$ family, we constructed the comparative syntenic maps of cucumber associated with Arabidopsis and tomato, respectively (Fig. 3b). Lots of $C s b H L H$ genes showed syntenic relationship with those in tomato and Arabidopsis, respectively. Interestingly, some $\mathrm{Cs} b \mathrm{HLH}$ genes were found to be associated with at least two syntenic gene pairs between cucumber and tomato, such as CsbHLHO24, CsbHLHO4O and CsbHLHO54 and so on. Similarly, there were also some $C s b H L H$ genes corresponding to two syntenic gene pairs between cucumber and Arabidopsis, for instance CsbHLHO2O and CsbHLHO49, indicating that these genes may have played an important role of $b H L H$ gene family during evolution. In addition, some collinear pairs (with CsbHLH132, CsbHLH135 and CsbHLH136, etc.) were identified between cucumber and both Arabidopsis and tomato (Fig. 3b; Table S2), indicating that these orthologous pairs may already exist before the ancestral divergence. Besides, we also found some $C s b H L H$ genes were not associated with syntenic gene pairs in Arabidopsis or tomato, indicating they may be peculiar to cucumber in the course of evolution.

\section{Ciselement analysis in the promoter regions of the CsbHLH genes in cucumber}

Previous studies have shown that many $b H L H$ genes play important roles in response to various abiotic stresses [29]. To analyze the putative functions of $C s b H L H$ genes in response to abiotic stresses, we identified and analyzed the promoter regions 2-kb 5'flanking region upstream from the start codon of each $\mathrm{CsbHLH}$ gene using PlantCARE software (Table S3), which most CsbHLH genes particularly presented the elements related to stress responsiveness (anaerobic induction, low temperature and drought inducibility) and plant hormones (Abscisic acid, Auxin, Gibberellic acid, Methyl jasmonate and Salicylic acid). Meanwhile, the promoter regions of some $C s b H L H$ genes had MYB binding site involved in flavonoid biosynthetic genes regulation, which maybe involved in the synthesis of flavonoid in cucumber (Fig. 4; Table S3). In addition to the stress and hormone responsive elements, the CsbHLH genes promoter regions also contained many light response elements (such as G-Box and Box-4). In general, The cis-regulatory elements present within $C s b H L H$ promoters could be divided into three major categories. The first category was the plant light-responsive elements (such as G-box, AE-box and I-box), the second kind included plant growth- and development-responsive elements (such as 02-site and ARE). The third category included elements that respond to diverse stresses (such as TC-rich, ABRE, MBS and LTR), which were widely distributed throughout the $C s b H L H$ gene family (Table S3).

\section{Function prediction of CsbHLHs based on phylogenetic analyses}


In Arabidopsis, the functions of many bHLH proteins have been characterized and verified [30, 31]. However, little is known about the biological functions of CsbHLHs in cucumber. In this study, we used phylogenetic analyses based on the protein of $142 \mathrm{CsbHLHs}$ and 166 AtbHLHs to identify putative orthologous and paralogous bHLH proteins in cucumber and Arabidopsis, which could preliminarily predicte the functions of CsbHLH proteins $[2,10]$ (Fig. 5).

In all, 23 subfamilies were clustered, and the functions of CsbHLHs were predicted based on their homologs with verified functions in the same cluster (Table S4). As shown in Table S4, the most of the members of subfamilies 1,2, 4, 10,13,14 and 18 response to diverse abiotic and biotic stresses [32, 33], including cold [34, 35], salt [36], and drought [37]. Some proteins in subfamilies 4 and 10 may be related to iron regulation, modulating the homeostasis of iron content [38]. Members of subfamilies 19 and 23 were predicted to regulate flower development [39], while the members of subfamilies 3, 8, 9, 16 and 21 may be involved in the development of various plant organs $[40,41,42]$. There were PIFs in the subfamily 17 , which are related with light signaling transduction and protect the normal growth and development of plants [15]. The members of subfamily 5 regulate the flavonoid biosynthesis and cell differentiation of root epidermis [24]. The members of subfamily 12 positively modulate the shade avoidance syndrome in Arabidopsis seedlings and are involved in brassinosteroid signaling [43]. The detailed possible functions of CsbHLHs are listed in Table S4.

In general, although evolutionary relationship could not be clearly deciphered for the relationships and functions of all genes, the analysis were meaningful and necessary.

\section{Expression analysis of the CsbHLH genes under different stress conditions and in different tissues}

To identify which of these $C s b H L H$ genes are most important in the response to abiotic stresses, we carefully screened 21,20 and 25 bHLH genes based on the cis-acting elements containing low temperatures, defense and stress responsive and Abscisic acid (ABA) on the promoters of bHLH genes, respectively, and detected their transcriptional changes in $4^{\circ} \mathrm{C}$ low temperature, salt $(\mathrm{NaCl})$ and $A B A$ treatment, respectively. As expected, all of the $C s b H L H$ genes screened responded to stress treatments under the respective stress conditions (Fig. 6). For example, the expression levels of the $20 \mathrm{CsbHLHs}$ all positive responsed to salt stress, and most were upregulated after an hour of treatment and achieved their maximum values after $3 \mathrm{~h}$ of treatment, decreasing thereafter. The expression levels of CsbHLHO2O, CsbHLH086 and CsbHLH109, were upregulated initially and kept going up untill reaching their maximum after $3 \mathrm{~h}$ of treatment, and then downregulated. The expression levels of CsbHLHO33, CsbHLHO41 and CsbHLH082 reached a maximum after just $1 \mathrm{~h}$ of $\mathrm{NaCl}$ treatment, while the expression levels of CsbHLH136 were highest after $12 \mathrm{~h}$. It could be seen from the results that $C s b H L H 041$ was the most susceptible to salt stress (increased by about 37-fold) (Fig. 6a). 
Under ABA treatment, the expression levels of most of the $25 \mathrm{CsbHLH}$ genes were up-regulated at different time points. Among them, CsMYC1, CsbHLH086, CsbHLH087, CsbHLH112, CsbHLH117, CsbHLH135 and $\mathrm{CsbHLH142}$ reached their highest expression levels after $3 \mathrm{~h}$ of ABA treatment. We also found only the expression level of CsbHLHO2O, CsbHLHO41 and CsbHLHO64 increased more than 10-fold compared to its untreated level ( $\mathrm{CsbHLH020}$ : the highest nearly 61-fold; $C s b H L H 041$ : the highest nearly 55-fold; CsbHLH064: the highest nearly 19-fold). In contrast, the expression levels of four of the CsbHLHs genes were significantly down-regulated under the ABA treatment (CsbHLH011, CsbHLHO33, CsbHLHO34 and CsbHLHO77), as could be seen from Fig. 6b.

The expression levels of 20 of the $21 \mathrm{CsbHLH}$ were up-regulated at some time points after $4^{\circ} \mathrm{C}$ treatment, while only $\mathrm{CsbHLHO32}$ was decreased under $4^{\circ} \mathrm{C}$ treatment (Fig. 6c). We could found that the 20 $\mathrm{Cs} b H L H s$ genes were upregulated at one or two time points,but none of them were upregulated at each time point. It could be seen from Fig. 6 that CsbHLH02O, CsbHLHO64, CsbHLH086, CsbHLHO93 and CsbHLH112 genes could simultaneously respond to the three abiotic stresses.

The expression patterns of genes under different conditions are often related to their functions. Therefore, qRT-PCR were used to detect the expression patterns for CsbHLH020, CsbHLH064, CsbHLH086, CsbHLH093 and CsbHLH112 abiotic stress-responsive CsbHLHgenes in the roots, stems, young leaves, male flowers, ovaries and tendrils. The expression patterns of the five $C s b H L H$ genes showed different tissue specificity (Fig. 6d). For example, CsbHLHO93 and CsbHLH112 showed high levels of transcript abundance in roots, and ovaries, but low levels in the male flowers and tendrils (Fig. 6d). In contrast, both CsbHLHO64 and CsbHLH086 showed high expression levels in the male flowers and tendrils. The expression levels of $\mathrm{CsbHLHO2O}$ was higher in the roots and young leaves than in other tissues (Fig. 6d). These results suggested that cucumber $b H L H$ genes might be involved in many aspects of physiological and developmental processes.

\section{CsbHLH041 enhanced tolerance to $\mathrm{NaCl}$ and $\mathrm{ABA}$ in transgenic Arabidopsis and cucumber}

CsbHLH041 expression was significantly induced by salt and ABA in cucumber (Fig. 6a-6b), therefore, we used Agrobacterium-mediated transient transformation of cucumber cotyledons to clarify CsbHLHO41 tolerance to salt and ABA. After $0.5 \mathrm{~h}$ of $100 \mathrm{mM} \mathrm{NaCl}$ treatment in hydroponic culture, serious wilting occurred in the seedings overexpressing 35 S empty vector compared with over-expression CsbHLHO41, and the wilting difference was more obvious after 1 hour of $\mathrm{NaCl}$ treatment, which showed overexpression CsbHLHO41 significant salt resistance (Fig. 7a). Under the ABA treatment, the CsbHLHO41 transgenic cucumber seedings proved more vigorous than the 35S empty vector (Fig. 7b). After 6 hour of ABA stress, 35 cucumber seedings showed visible symptoms of ABA-induced damage, such as drying, wilting, and even death after treatment for 12 hour, while some $C s b H L H 041$ transgenic plants remained green with expanded cotyledons (Fig. 7b). 


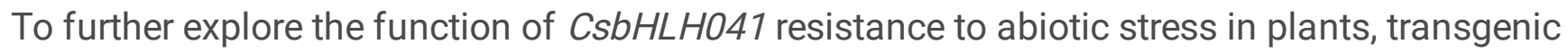
Arabidopsis plants overexpressing CsbHLHO41 driven by the CaMV35S promoter were generated. Two independent homozygous lines CsbHLH041 OX-1 and CsbHLH041 OX-2 with relatively high expression

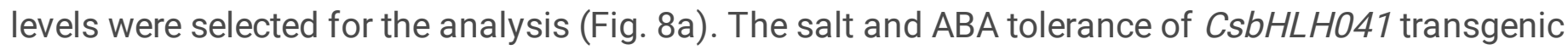
plants was assessed. There were no differences in the seeds germination between WT and CsbHLHO41 transgenic Arabidopsis on 1/2 MS (Control) (Fig. 8b). However, only about 15-18\% of the WT seeds germinated in $1 / 2 \mathrm{MS}$ medium containing $100 \mathrm{mM} \mathrm{NaCl}$ or $2 \mu \mathrm{M} \mathrm{ABA}$, while about $70-80 \%$ of CsbHLHO41 transgenic plants seeds were able to germinate (Fig. 8b-d). In a word, the germination ratio of transgenic plants seeds was remarkably higher than that of WT seeds in the 1/2 MS medium containing $100 \mathrm{mM} \mathrm{NaCl}$ or $2 \mu \mathrm{M} \mathrm{ABA}$ (Fig. 8b-d). Subsequently, the 3-week-old seedlings of CsbHLH041 transgenic

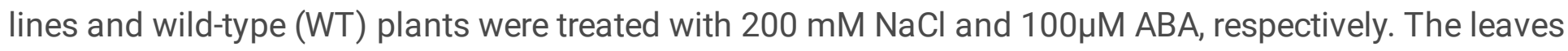
of WT plants turned yellow severely after 4 days of $200 \mathrm{mM} \mathrm{NaCl}$ or $100 \mu \mathrm{M}$ ABA treatment, while with regard to $C s b H L H 041$ transgenic lines still growing with green leaves (Fig. 8e-f). After 8 days, the difference of $\mathrm{NaCl}$ or ABA resistance between the WT plants and CsbHLH041 transgenic lines was more obvious, which suggested that $C s b H L H 041$ transgenic plants were more tolerant to salt and ABA stresses than WT plants.

\section{The protein interaction network predictions for CsbHLH orthologs in Arabidopsis that were crucial tothe abiotic stress response}

Network interaction analysis has been demonstrated to be an effective method to analyze the gene function [44]. We used online software of STRING 10 to predict protein interaction network with the 142 $\mathrm{CsbHLH}$ protein sequences as queries, which formed a complex interaction network constructed with orthologs in Arabidopsis. Lots bHLH proteins interacted with more than one bHLH (Fig. 9a), which was consistent with previous reports that the binding activity of specific DNA sequences depended on the formation of homodimers or heterodimers of different bHLH proteins [2]. In all, 21 proteins were showed that could interact with more than four other bHLH proteins, which maked them important players in regulating plant growth and stress responses, and detailed information about these orthologs was also summarized in Table S6.

We examined that CsbHLH041 showed a significant response to salt and ABA treatments (Fig. 6a-6b),

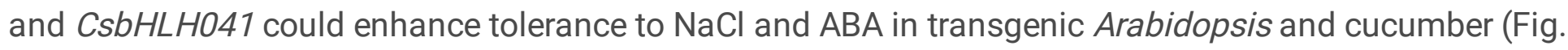
7; Fig. 8). The bHLH proteins function mainly by forming homodimers or heterodimers with other proteins, which are essential for their binding to related downstream genes [2]. The CsbHLHO41 homologous gene, AT5G56960, was located in the center of the predicted gene association network, which showed it played main roles in regulating various proteins with different functions (Fig. 9b; Table S6). For example, EP3 might play a role in both normal plant growth and disease resistance [45]. VSP1 and VSP2 were antiinsect protein and also response to Methyl Jasmonate and wounding, which their defense function were 
correlated with its acid phosphatase activity $[46,47]$. The predicted gene association network provides useful resources for subsequent research.

\section{Discussion}

\section{Characterization of the cucumber $b H L H$ family}

The basic helix-loop-helix (bHLH) transcription factor family is the second largest in eukaryotes [10, 48] and extensive studies of the $b H L H$ families have been identified in various plants [2]. For example, 166 $b H L H$ genes have been identified in Arabidopsis [2, 10], 115 bHLH genes in Nelumbo nucifera [49], 188 $b H L H$ genes in apple [44], $167 \mathrm{bHLH}$ genes in rice [12], $94 \mathrm{bHLH}$ genes in grape [50], $113 \mathrm{bHLH}$ genes in strawberry [51] and $159 \mathrm{bHLH}$ genes in tomato [13]. The bHLH transcription factors have been involved in multiple biological processes in plants, especially in regulating defense against biotic and abiotic stresses [52]. However, very little information is available about the $b H L H s$ in cucumber. In our study, 142 $b H L H$ cucumber genes were identified and characterized based on the published cucumber annotated genes in cucumber genome through genome-wide analysis, which were further classified into 32 subgroups based on phylogenetic analyses (Fig. 2a). Chromosomal localization analysis revealed that the $\mathrm{Cs} b \mathrm{HLH}$ genes were distributed in seven chromosomes of cucumber with different density and number. Only one gene could not be pinpointed on any of the chromosomes, which might be due to the current genome incomplete database of cucumber. Therefore, more detailed cucumber genomic data in the future would help pinpoint the gene. Multiple sequence alignment of the full-length CsbHLH protein sequences showed that the $142 \mathrm{CsbHLH}$ contained the conserved bHLH domain, and the number and ratio of the conserved amino acid were similar to the Arabidopsis (Fig. 1). For example, the two amino acid residues Leu-21 and Leu-53 were relatively conserved in the helical region that determined whether a dimeric complex could be formed. Moreover, the conserved motif analyses showed that almost all the CsbHLH family members had the 1 and 2 motif representing the conserved bHLH domain, and the analysis of motif and gene structure were further performed to gain evidence to support phylogenetic relationship for $142 \mathrm{CsbHLH}$ gene family (Fig. 2b-2c). In summary, these results indicated that the 142 $C s b H L H s$ all contained characteristics of the $b H L H$ family, confirming the accuracy of $b H L H$ gene family detection in cucumber.

\section{Phylogenetic analysis and evolution of cucumber $b H L H$ Genes}

Considering the model plant Arabidopsis has systematically analyzed the $b H L H$ gene family $[2,11]$. To explore the evolutionary relationships between $142 \mathrm{CsbHLH}$ proteins in cucumber and $166 \mathrm{AtbHLH}$ proteins in Arabidopsis, a phylogenetic tree was constructed based on the protein of $308 \mathrm{bHLHs}$, which clustered into 23 subfamilies (Fig. 5). There are differences in anatomy and physiology between cucumber and Arabidopsis, so some clades may have different ways of expansion in the $b H L H$ family of cucumber and Arabidopsis. From Fig. 5 and Table S4 known, not all bHLH members in cucumber were 
included in these 23 subfamilies, which suggested that there were differences between cucumber and Arabidopsis during the process of evolution.

Studies had shown that gene duplication events played a crucial role in the rapid expansion and evolution of gene families [28]. In cucumber genome, total 1468 tandem duplication gene pairs and 231 segmental duplication blocks were identified, respectively (Table S1). Seven segmental duplication events were identified in cucumber using MCScanX, and five tandem duplication gene pairs were obtained in CsbHLH family, respectively (Fig. 3a). In general, the gene functions of a clade is highly conserved among different plant species, but it is not absolute. Therefore, it is of great significance to accurately identify the true orthologs between plants species based on synteny analysis, so comparative syntenic analysis between cucumber, Arabidopsis and tomoto genomes was performed to explore the origin and evolutionary process of cucumber $b H L H$ genes. The results showed that the cucumber genome had extensive synteny with Arabidopsis genome and tomato genome, and 944 syntenic blocks and 983 syntenic blocks between cucumber and Arabidopsis and tomato genome were identified, respectively (Table S5). Many CsbHLH genes showed a linear relationship with the genes of tomato and Arabidopsis, respectively. We observed that some $C s b H L H$ genes were found to be associated with at least two syntenic gene pairs between cucumber and Arabidopsis and tomato, respectively. For example, the genes AT2G22750.3 and AT4G37850.2 were orthologs of CsaV3_6G044560 in Arabidopsis, and Solyc02g093280.2.1 and Solyc04g078690.2.1 were orthologs of CsaV3_6G043370 in tomato, which indicated that these genes might have played an important role of $b H L H$ gene family during evolution (Fig. 3b; Table S2), indicating that these orthologous pairs might already exist before the ancestral divergence.

It has been previously suggested that orthologous genes usually have similar functions and are clustered in the same clade and subclades. As shown in Fig. 5, lots cucumber bHLH proteins were clustered into some Arabidopsis functional clades, which provided valuable information for studying the function of cucumber bHLHgenes. CsaV3_6G000530 (CsMYC1) and CsaV3_6G037080 (CsbHLH042) were grouped into subfamily5 along with AtGL3, AtEGL3, AtMYC1 and AtTT8, and highly homologous to these proteins. In Arabidopsis, AtGL3, AtEGL3 and AtTT8 have been demonstrated as being the key regulators of anthocyanin and PA biosynthesis [24]. Meanwhile, AtGL3, AtEGL3 and AtMYC1 were shown to regulate trichome formation and root hair patterning $[19,53]$. Therefore, it is possible that CsMYC1 and CsbHLH042 may control trichome formation and PA biosynthesis in cucumber. However, this needs further investigation. In addition, other homologues of AtMYC1, for example, VvMYC1 has been shown to control anthocyanin and PA biosynthesis [54], which further proves that high homologous proteins have similar functions.

\section{Cucumber $b H L H$ genes may play important roles in abiotic stress tolerance}


In the process of plant response to abiotic stress, bHLH transcription factors act as regulatory genes to regulate the expression changes of related stress genes, thus playing an important role in stress responses. Many studies have shown that bHLH TFs can respond to a range of stresses. For example, in addition to being involved in the morphogenesis of stomata, the TFs INDUCER OF CBF EXPESSION1 (ICE1) and ICE2 in Arabidopsis and their homologous genes in other species can play key roles in the response to low temperature stress $[34,52]$. RERJ1 is upregulated in the event of physical damage and drought stress to plants [55]. In terms of salt tolerance, the overexpression of AtbHLH92 gene in Arabidopsis can significantly enhance the tolerance of plants to salt damage and osmotic stress [56]. All these examples indicate that bHLH TFs can play a certain role in response to abiotic stress. However, little is known about the functions of the $b H L H$ gene family in cucumber. To better analyze the protein functions of the $b H L H$ gene family in cucumbers, we conducted preliminary analysis of three aspects to reveal the functions of the $C s b H L H$ gene family.

How cis-elements in the promoters of the $b H L H$ genes respond to the environment will affect their roles in stimulating and regulating gene expression. Cis-element analyses showed that elements that might respond to diverse stresses (such as LTR, TCA-element and MBS) were widely distributed in the CsbHLH gene family (Fig. 4). Most cucumber $b H L H$ gene promoters contained MYB binding sites, which are involved in drought response (Table S3). This reflected that they could be regulated by MYB transcription factors under drought stress. Some cucumber $b H L H$ gene promoters contained MYB binding site involved in flavonoid biosynthetic genes regulation, indicating that they might regulate flavonoid biosynthesis. Many cucumber $b H L H$ gene promoters contained ABRE and TC-rich elements, which are involved in ABAdependent or independent stress tolerance [57]. Therefore, according to the cis-acting element contained on the promoter, these genes may play important roles in gene regulation in response to different stresses in cucumber. In addition, the functions of $50 \mathrm{CsbHLHs}$ were predicted based on their known and verified homologs in Arabidopsis by phylogenetic tree between cucumber and Arabidopsis (Table S4), which were mainly associated with development processes (root hair development, stomatal development, seed dormancy, flower and fruit development) and stress responses (response to salicylic acid stimulus, wound, insect, drought, low-temperature and salt stress) (Table S4). The third aspect, we also used Arabidopsis orthologs to predict the regulatory networks for $142 \mathrm{CsbHLH}$ genes, which the results suggested that many member genes could respond to stimuli (Table S6). For example, bHLH093 and ICE1 were involved in ABA signaling pathway, which were crucial for abiotic stress response in plants $[57,58]$. All these analyses suggest that the $b H L H$ gene family may be also related to plant development, metabolic regulation, and the response to stress in cucumber, which were consistent with previous researchs $[10,12]$. Subsequently, we analyzed and screened $C s b H L H$ genes that might respond to stress, as it is very important to improve stress tolerance of cucumber. According to cis-element analyses, TCrich cis-elements that may be involved in defense and stress responses were detected in the promoter regions of $60 \mathrm{CsbHLH}$ genes (Fig. 4). Meanwhile, the promoters of $41 \mathrm{CsbHLHs}$ contained LTR element, which responds to cold stress and $106 \mathrm{CsbHLHs}$ contained ABA responsive element, which responds to ABA stress. The phylogenetic analyses between cucumber and Arabidopsis further showed that 25 CsbHLHs might respond to abiotic stresses, including cold, ABA, salt and drought basis for their 
homologs in Arabidopsis (Table S4). Through comprehensive analysis, we carefully screened 21, 20 and $25 \mathrm{bHLH}$ genes were likely to respond to $4^{\circ} \mathrm{C}$ low temperature, salt $(\mathrm{NaCl})$ and $\mathrm{ABA}$, respectively. The screened $\mathrm{CsbHLH}$ genes were all responded to stress treatments under the respective stress conditions (Fig. 6). We also found that CsbHLH020, CsbHLH064, CsbHLH086, CsbHLH093 and CsbHLH112 genes could simultaneously respond to low temperature, salt and ABA of three abiotic stresses (Fig. 6), which was preliminarily predicted that they might be involved in abiotic stress process. CsbHLHO41 was induced by salt and ABA (Fig. 6a-b), and the 35S:CsbHLH041 transgenic Arabidopsis thaliana and transient transformed cucumber cotyledons were proved to enhance salt and ABA resistance (Fig. 7; Fig. 8).The results of these analyses indicated that functional prediction of the $\mathrm{Cs} b \mathrm{HLH}$ gene family could provide valuable reference data for further functional studies of this gene family in cucumber.

\section{Conclusions}

Our study investigated the $b H L H$ family genes in detail in cucumber. We also performed expression analyses of the selected genes under different stress treatments, and detailed functions of CsbHLHO41 using the transgenic method. This work would provide abundant insights into the functions and regulatory mechanisms of CsbHLH proteins in cucumber abiotic stress tolerance and growth and development.

\section{Methods}

\section{Genome-wide identification of the CsbHLH genes in Cucumber}

To identify the $C s b H L H$ gene family members from the entire cucumber genome database, 166 Arabidopsis bHLH proteins were used as query sequences and Blastp searches against the predicted cucumber proteins. In addition, the Hidden Markov Model (HMM) profile of the bHLH domain (PF00010) from the Pfam database (available online: http://pfam.janelia.org) was also applied as a query to search the $b H L H$ genes from the cucumber genome database. All candidate genes were further examined the bHLH domains by using the Pfam and Simple Modular Architecture Research Tool (SMART) program (http://smart.embl-heidelberg.de) [26].

\section{Multiple sequence alignment and phylogenetic analysis}

Multiple sequence alignments were performed using ClustalX 1.81 with default parameters and DNAMAN software (http://dnaman.software.informer.com/). The sequence logos for bHLHs were obtained by submitting the multiple alignment sequences to the website submitting the multiple alignment sequences to the website (http://weblogo.berkeley.edu/logo.cgi) [59]. A phylogenetic tree was constructed with the aligned fully predicted protein sequences of $142 \mathrm{bHLH}$ genes using MEGA7 (https://www.megasoftware.net/) [60]. The neighbor-joining(NJ) method was used with the following 
parameters: Poisson correction, pairwise deletion, and bootstrap (1,000 replicates; random seed). The phylogenetic tree was visualized by plotting it using the EvolView tool (http://www.evolgenius.info). Classification of the $\mathrm{CsbHLH}$ genes was then performed according to their phylogenetic relationships with their corresponding Arabidopsis bHLHgenes.

\section{Gene structure and conserved motif analysis}

The DNA and cDNA sequences corresponding to each predicted gene from the cucumber genome were downloaded, and then the $142 \mathrm{Cs} b H L H$ gene structures were analyzed using the web-based bioinformatics tool GSDS (http://gsds.cbi.pku.edu.cn/) [61]. Conserved motif structures in CsbHLHs were identified using the MEME (http://meme-suite.org/index.html) [28].

\section{Chromosomal distribution and gene duplication}

All CsbHLHgenes were mapped to cucumber chromosomes based on physical location information from the database of cucumber genome using TBtools [28]. The gene duplication events was conducted using the Multiple Collinearity Scan toolkit (MCScanX), with the default parameters [62]. To identify the synteny relationship of the orthologous $b H L H$ genes obtained from cucumber, Arabidopsis and tomato, the syntenic analysis maps were constructed using TBtools [28].

\section{Analysis of the $b H L H$ genes promoter in cucumber}

We downloaded the entire cucumber genome sequence from the cucumber genome database (Chinese Long 9930) and extracted the 2-kb long sequences upstream of the transcription start site of these 142 $\mathrm{CsbHLH}$ genes. The cis-acting elements on the promoter regions of these genes were analyzed using PlantCARE (http://bioinformatics.psb.ugent.be/ webtools/ plantcare/ html/) software [63].

\section{Plant materials and growth conditions}

Cucumber (Cucumis sativus L. cv 'Xintaimici') seeds, which was provided by Professor Chenxing Cao (Shandong Agricultural University), were germinated on moist filter paper in an incubator at $28^{\circ} \mathrm{C}$ for 1 day. The germinated seeds were sown into soil mixture in an ordinary illuminated incubator at Shandong Agricultural University. After 10 days, batches of 12 seedlings were transferred to a plastic tank filled with an aerated nutrient solution ( $\mathrm{pH}$ 6.0-6.5) containing: $\mathrm{Ca}$ (NO3)2: $3.5 \mathrm{mM}, \mathrm{KNO}: 7 \mathrm{mM}, \mathrm{KH} 2 \mathrm{PO} 4: 0.78$ mM, MgSO4: 2 mM, H3B03: $29.6 \mu \mathrm{M}$, MnSO4: $10 \mu \mathrm{M}$, Fe-EDTA: $50 \mu \mathrm{M}$, ZnS04: $1.0 \mu \mathrm{M}$, H2Mo04: $0.05 \mu \mathrm{M}$ and CuSO4: $0.95 \mu \mathrm{M}$ ( $\mathrm{Li}$ et al. 2012a, b). The experiment was carried out in an illuminated incubator and the air temperature $\left(25^{\circ} \mathrm{C}\right.$ during the day and $18^{\circ} \mathrm{C}$ during the night) and light intensity ( $400 \mu \mathrm{mol}$ $\mathrm{m}^{-2} \mathrm{~s}^{-1}$ ) regimes were maintained throughout each treatment. When the cucumber seedlings were at the three-true-leaf stage, three treatments were conducted, respectively: $100 \mathrm{mM} \mathrm{NaCl}, 100 \mu \mathrm{M} \mathrm{ABA}, 4^{\circ} \mathrm{C}$. 
Leaves for RNA extraction were harvested at $0,0.5,1,3,6,12$ and $24 \mathrm{~h}$ after the three treatments, respectively, immediately frozen in liquid nitrogen and stored at $-80^{\circ} \mathrm{C}$ for subsequent analyses. The $\mathrm{NaCl}$ was added into nutrient solution and ABA was sprayed onto the leaves. The roots, stems, leaves, male flowers, fruits and tendrils of mature plants were collected separately and used for tissue-specific expression analysis. Each expression profile was independently verified in three replicate experiments performed under identical conditions and 15 cucumber seedlings were used for each replicate experiment.

\section{RNA extraction and analysis by qRT-PCR}

All plant material was collected, frozen in liquid nitrogen, and extracted under RNase-free conditions. The RNA was extracted with TRizol reagent (Invitrogen, Carlsbad, CA, USA), according to the manufacturer's instructions. One $\mu \mathrm{g}$ of total RNA was used to synthesize cDNA with the PrimeScript ${ }^{\circledR} 1$ st Strand cDNA Synthesis Kit (Takara, Japan), according to the manufacturer's instructions. QRT-PCR was performed using the SYBR green detection protocol (Takara) with an Applied Biosystems 7500 real-time PCR system (Applied Biosystems). The analysis of relative mRNA expression data was performed using the $2^{-} \triangle \triangle \mathrm{Ct}$ method [64], and expression data were normalized to the $\beta$-actin gene. Each expression profile was independently verified in 3 replicate experiments performed under identical conditions. The heatmaps were generated using TBtools [28]. All primers used in this study are listed in Table S7.

\section{Overexpression vector construction, Arabidopsis transformation and transient transformation in cucumber cotyledons}

The full-length coding sequence of $C s b H L H 041$ was recombined into the pCAMBIA1300 vector. The primers used are listed in Table S7. The construct was transformed into Agrobacterium tumefaciens LBA4404, which was used for transformation of Arabidopsis plants by the floral dip method. The Arabidopsis seeds was Colombia (Col-0), which was bred in our laboratory. Homozygous T3 transgenic Arabidopsis lines were identified by hygromycin $(300 \mathrm{mg} / \mathrm{L})$ selection, which was then treated with different abiotic stresses. After Agrobacterium tumefaciens LBA4404 was shaken to an OD600 value of $0.6-0.8$, cells were harvested by centrifugation and resuspended in MES suspension liquid [65]. The Agrobacterium suspension was then infiltrated into cotyledons of 8-d-old cucumber seedlings [65] using a needleless syringe. After 2 days, the samples were treated with different abiotic stresses and the phenotype was observed at different time periods.

\section{Abiotic stress tolerance assays and ABA sensitivity analysis}


For Arabidopsis salt stress and ABA treatment, the seeds of CsbHLH041 T3-generation homozygous lines and the Col-0 (WT) were sown on vermiculite soil in pots and cultured under normal conditions at $22^{\circ} \mathrm{C}$ for 3 weeks. For salt treatment, the 3-week-old seedlings were watered with $200 \mathrm{mM} \mathrm{NaCl}$ solution every other day and the growing of Col-O (WT) and CsbHLHO41 transgenic lines was observed every 4 days. For ABA treatments, the 3-week-old seedlings were watered with $100 \mu \mathrm{M}$ ABA solution every other day and phenotypes were also measured every 4 days. To check seed germination rate to salt stress and ABA treatment, the seeds of Col-0 (WT) and transgenic lines were surface sterilized and sown on 1/2 MS medium supplemented with $2 \mu \mathrm{M} \mathrm{ABA}$ or $100 \mathrm{mM} \mathrm{NaCl}$, respectively, under normal conditions at $22^{\circ} \mathrm{C}$ in a growth chamber. The germination rate was scored on the 7th day after culturing on the plates.

For determining the salt tolerance and ABA sensitivity in cotyledons of 8-d-old cucumber seedlings with transient infiltration of $35 \mathrm{~S}$ and $35 \mathrm{~S}: \mathrm{Cs} b \mathrm{HLH041}$, the select seedlings with the same growth were transfered to $6 \mathrm{~L}$ nutrient solution for hydroponics. Hoagland nutrient solution was used for culture, and the seedlings were grown hydroponically for two days before salt and ABA treatment. Then the seedlings were treated with salt and $A B A$, and the final concentration in the medium was $100 \mathrm{mM}$ and $100 \mu \mathrm{M}$, respectively. In order to ensure the reliability of the experiment, the cucumber seedlings with transient infiltration of $35 S$ and $35 S$ : CsbHLH041 were cultured in the same hydroponic box. The changes of transgenic and control seedlings were observed at different time periods.

\section{Protein association network predictions and functional annotations by STRING}

The 142 CsbHLH protein sequences were submitted to the online server STRING (version 10.0; http://string-db.org)," with the organism specified as "Arabidopsis thaliana."' After the blast step was finished, genes with the highest bitscores were used to construct the network. The bHLHs that did not interact with any others were removed. The functional annotation information was copied manually from the blast results.

\section{Abbreviations}

bHLH, basic Helix-Loop-Helix; At, Arabidopsis thaliana; Cs, Cucumis sativus L; MS, Murashige and Skoog; qRT-PCR, quantitative reverse transcription-PCR; CDS, Coding Sequence; ABA, Abscisic acid; pl, isoelectric point; WT, wild type.

\section{Declarations}

\section{Ethics approval and consent to participate}

Not applicable. 


\section{Consent for publication}

Not applicable.

\section{Availability of data and materials}

The data that support the results are included within the article and its additional files. Other relevant materials are available from the corresponding authors on reasonable request.

\section{Competing interests}

The authors declare that they have no competing interests.

\section{Funding}

This work was supported by fundings from the National Natural Science Foundation of China (31672170 and 31872950), the Natural Science Foundation of Shandong Province (JQ201309), the Shandong "Double Tops" Program (SYL2017YSTD06) and the 'Taishan Scholar' Foundation of the People's Government of Shandong Province (ts20130932). The funds played no role in study design, data analysis, and manuscript preparation.

\section{Authors' contributions}

$\mathrm{JL}$ and ZR conceived and designed the experiments. JL, TW and JH performed the experiments. JL analyzed the data and wrote the manuscript. ZR revised the manuscript. All authors have read and approved this manuscript.

\section{Acknowledgments}

We thank Professor Chenxing Cao, College of Horticultural Science and Engineering, Shandong Agricultural University, for providing cucumber (Cucumis sativus L. cv 'Xintaimici') seeds.

\section{References}

1. Riechmann JL, Heard J, Martin G, Reuber L, Jiang C, Keddie J, et al. Arabidopsis transcription factors: genome-wide comparative analysis among eukaryotes. Science. 2000;290:2105-2110.

2. Carretero-Paulet L, Galstyan A, Roig-Villanova I, Martinez-Garcia JF, Bilbao-Castro JR, Robertson DL. Genome-wide classification and evolutionary analysis of the bHLH family of transcription factors in Arabidopsis, poplar, rice, moss, and algae. Plant Physiol. 2010;153 3):1398-1412. 
3. Ferré-D'Amaré AR, Prendergast GC, Ziff EB, Burley S K. Recognition by Max of its cognate DNA through a dimeric b/HLH/Z domain. Nature. 1993;363:38-45.

4. Murre $C$, Mccaw PS, Baltimore D. A new DNA binding and dimerization motif in immunoglobulin enhancer binding, daughterless, MyoD, and myc proteins. Cell. 1989;56:777-783.

5. Shimizu T, Toumoto A, Ihara K, Shimizu M, Kyogoku Y, Ogawa N, Oshima Y, Hakoshima T. Crystal structure of PHO4 bHLH domain-DNA complex: flanking base recognition. EMBO J. 1997;16:46894697.

6. Massari ME, Murre C. Helix-loop-helix proteins: regulators of transcription in eucaryotic organisms. Mol Cell Biol. 2000;20:429-440.

7. Nair SK, Burley SK. Recognizing DNA in the library. Nature. 2000; 404:715,717-718.

8. Atchley WR, Terhalle W, Dress A. Positional dependence, cliques, and predictive motifs in the bHLH protein domain. Journal of Molecular Evolution. 1999;48:501-516.

9. David C, Pierre K, Morgane TC, Gemma R, Valérie L, Elena S, Degnan BM, Michel V. Origin and diversification of the basic helix-loop-helix gene family in metazoans: insights from comparative genomics. BMC Evol. Biol. 2007;7:33.

10. Toledo-Ortiz G, Huq E, Quail PH. The Arabidopsis basic/helix-loop-helix transcription factor family. Plant Cell. 2003;15(8):1749-1770.

11. Heim MA, Jakoby M, Werber M, Martin C, Weisshaar B, Bailey PC. The basic helix-loop-helix transcription factor family in plants: a genome-wide study of protein structure and functional diversity. Mol Biol Evol. 2003;20(5):735-747.

12. Li X, Duan X, Jiang H, Sun Y, Tang Y, Yuan Z, et al. Genome-wide analysis of basic/helix-loop-helix transcription factor family in rice and Arabidopsis. Plant Physiol. 2006; 141(4):1167-1184.

13. Sun H, Fan HJ, Ling HQ. Genome-wide identification and characterization of the bHLH gene family in tomato. BMC Genomics. 2015;16:9.

14. Ludwig SR, Habera LF, Dellaporta SL, Wessler SR. Lc, a member of the maize R gene family responsible for tissue-specific anthocyanin production, encodes a protein similar to transcriptional activators and contains the myc-homology region. Proc Natl Acad Sci U S A. 1989;86:7092-7096.

15. Paik I, Kathare PK, Kim JI, Huq E. Expanding Roles of PIFs in Signal Integration from Multiple Processes. Mol Plant. 2017;10(8):1035-1046.

16. Lee S, Lee S, Yang KY, Kim YM, Park SY, Kim SY, et al. Overexpression of PRE1 and its homologous genes activates Gibberellin-dependent responses in Arabidopsis thaliana. Plant Cell Physiol. 2006;47(5):591-600.

17. Feyissa DN, Lovdal T, Olsen KM, Slimestad R, Lillo C. The endogenous GL3, but not EGL3, gene is necessary for anthocyanin accumulation as induced by nitrogen depletion in Arabidopsis rosette stage leaves. Planta. 2009;230(4):747-754.

18. Appelhagen I, Jahns O, Bartelniewoehner L, Sagasser M, Weisshaar B, Stracke R. Leucoanthocyanidin Dioxygenase in Arabidopsis thaliana: characterization of mutant alleles and 
regulation by MYB-BHLH-TTG1 transcription factor complexes. Gene. 2011;484(1-2):61-68.

19. Ramsay NA, Glover BJ. MYB-bHLH-WD40 protein complex and the evolution of cellular diversity. Trends Plant Sci. 2005;10(2):63-70.

20. Zhai Y, Zhang L, Xia C, Fu S, Zhao G, Jia J, et al. The wheat transcription factor, TabHLH39, improves tolerance to multiple abiotic stressors in transgenic plants. Biochem Biophys Res Commun. 2016;473(4):1321-1327.

21. Xu W, Zhang N, Jiao Y, Li R, Xiao D, Wang Z. The grapevine basic helix-loop-helix (bHLH) transcription factor positively modulates CBF-pathway and confers tolerance to cold-stress in Arabidopsis. Mol Biol Rep. 2014;41(8):5329-5342.

22. Grimault A, Gendrot G, Chamot S, Widiez T, Rabille H, Gerentes MF, et al. ZmZHOUPI, an endospermspecific basic helix-loop-helix transcription factor involved in maize seed development. Plant $\mathrm{J}$. 2015;84(3):574-586.

23. Fernández-Calvo P, Chini A, Fernandez-Barbero G, Chico JM, Gimenez-Ibanez S, Geerinck J, et al. The Arabidopsis bHLH transcription factors MYC3 and MYC4 are targets of JAZ repressors and act additively with MYC2 in the activation of jasmonate responses. Plant Cell. 2011;23(2):701-715.

24. Li S. Transcriptional control of flavonoid biosynthesis. Plant Signaling \& Behavior. 2014;9:e27522.

25. Huang S, Li R, Zhang Z, Li L, Gu X, Fan W, et al. The genome of the cucumber, Cucumis sativus Nat Genet. 2009;41(12):1275-1281.

26. Letunic I, Doerks T, Bork P. SMART 6: recent updates and new developments. Nucleic Acids Res 37 (Database issue). 2009;D229-232.

27. Cheng X, Xiong R, Liu H, Wu M., Chen F, Hanwei Y, Xiang Y. Basic helix-loop-helix gene family: Genome wide identification, phylogeny, and expression in Moso bamboo. Plant Physiol Biochem. 2018;132:104-119.

28. Sun H, Pang B, Yan J, Wang T, Wang L, Chen C, et al. Comprehensive Analysis of Cucumber Gibberellin Oxidase Family Genes and Functional Characterization of CsGA20ox1 in Root Development in Arabidopsis. Int J Mol Sci. , 2018;19(10).

29. Babitha KC, Vemanna RS, Nataraja KN, Udayakumar M. Overexpression of EcbHLH57 Transcription Factor from Eleusine coracana L. in Tobacco Confers Tolerance to Salt, Oxidative and Drought Stress. PLoS One. 2015;10(9):e0137098.

30. Abe H, Urao T, Ito T, Seki M, Shinozaki K, Yamaguchi-Shinozaki K. Arabidopsis AtMYC2 (bHLH) and AtMYB2 (MYB) function as transcriptional activators in abscisic acid signaling. Plant Cell. 2003;15(1):63-78.

31. Petridis A, Doll S, Nichelmann L, Bilger W, Mock HP. Arabidopsis thaliana G2-LIKE FLAVONOID REGULATOR and BRASSINOSTEROID ENHANCED EXPRESSION1 are low-temperature regulators of flavonoid accumulation. New Phytol. 2016;211(3):912-925.

32. Sasaki-Sekimoto Y, Saito H, Masuda S, Shirasu K, Ohta H. Comprehensive analysis of protein interactions between JAZ proteins and bHLH transcription factors that negatively regulate jasmonate signaling. Plant Signal Behav. 2014;9(1):e27639. 
33. Song S, Qi T, Fan M, Zhang X, Gao H, Huang H, et al. The bHLH subgroup Illd factors negatively regulate jasmonate-mediated plant defense and development. PLoS Genet. 2013;9(7):e1003653.

34. Chinnusamy V, Ohta M, Kanrar S, Lee BH, Hong X, Agarwal M, Zhu JK. ICE1: a regulator of coldinduced transcriptome and freezing tolerance in Arabidopsis. Genes Dev. 2003;17(8):1043-1054.

35. Kashyap P, Deswal R. Two ICE isoforms showing differential transcriptional regulation by cold and hormones participate in Brassica juncea cold stress signaling. Gene. 2019;695:32-41.

36. Ahmad A, Niwa Y, Goto S, Ogawa T, Shimizu M, Suzuki A,et al. bHLH106 Integrates Functions of Multiple Genes through Their G-Box to Confer Salt Tolerance on Arabidopsis. PLoS One. 2015;10(5):e0126872.

37. Le Hir R, Castelain M, Chakraborti D, Moritz T, Dinant S, Bellini C. AtbHLH68 transcription factor contributes to the regulation of $\mathrm{ABA}$ homeostasis and drought stress tolerance in Arabidopsis thaliana. Physiol Plant. 2017;160(3):312-327.

38. Kurt F, Filiz E. Genome-wide and comparative analysis of $b H L H 38, b H L H 39, b H L H 100$ and $b H L H 101$ genes in Arabidopsis, tomato, rice, soybean and maize: insights into iron (Fe) homeostasis. Biometals. 2018;31(4):489-504.

39. Sharma N, Xin R, Kim DH, Sung S, Lange T, Huq E. NO FLOWERING IN SHORT DAY (NFL) is a bHLH transcription factor that promotes flowering specifically under short-day conditions in Arabidopsis. Development. 2016;143(4):682-690.

40. Karas B, Amyot L, Johansen C, Sato S, Tabata S, Kawaguchi M, et al. Conservation of lotus and Arabidopsis basic helix-loop-helix proteins reveals new players in root hair development. Plant Physiol. 2009;151(3):1175-1185.

41. Yang KZ, Jiang M, Wang M, Xue S, Zhu LL, Wang HZ, et al. Phosphorylation of Serine 186 of bHLH Transcription Factor SPEECHLESS Promotes Stomatal Development in Arabidopsis. Mol Plant. 2015;8(5):783-795.

42. Chakraborty M, Gangappa SN, Maurya JP, Sethi V, Srivastava AK, Singh A, et al. Functional interrelation of MYC2 and HY5 plays an important role in Arabidopsis seedling development. Plant $\mathrm{J}$. 2019;99(6):1080-1097.

43. Zhang LY, Bai MY, Wu J, Zhu JY, Wang H, Zhang Z, et al. Antagonistic HLH/bHLH transcription factors mediate brassinosteroid regulation of cell elongation and plant development in rice and Arabidopsis. Plant Cell. 2009;21(12):3767-3780.

44. Mao K, Dong Q, Li C, Liu C, Ma F. Genome Wide Identification and Characterization of Apple bHLH Transcription Factors and Expression Analysis in Response to Drought and Salt Stress. Front Plant Sci. 2017;8:480.

45. Passarinho PA, Van Hengel AJ, Fransz PF, de Vries SC. Expression pattern of the Arabidopsis thaliana AtEP3/Atchit/V endochitinase gene. Planta. 2001;212(4):556-567.

46. Berger S, Bell E, Mullet JE. Two Methyl Jasmonate-Insensitive Mutants Show Altered Expression of AtVsp in Response to Methyl Jasmonate and Wounding. Plant Physiol. 1996;111:525-531. 
47. Chi YH, Jing X, Lei J, Ahn JE, Koo YD, Yun DJ, et al. Stability of AtVSP in the insect digestive canal determines its defensive capability. Journal of Insect Physiology. 2011;57(3):391-399.

48. Ledent $\mathrm{V}$, Vervoort $\mathrm{M}$. The basic helix-loop-helix protein family: comparative genomics and phylogenetic analysis. Genome Res. 2001;11:754-770.

49. Mao TY, Liu YY, Zhu HH, Zhang J, Yang JX, Fu Q, et al. Genome-wide analyses of the bHLH gene family reveals structural and functional characteristics in the aquatic plant Nelumbo nucifera. 2019;7:e7153.

50. Wang P, Su L, Gao H, Jiang X, Wu X, Li Y, et al. Genome-Wide Characterization of bHLH Genes in Grape and Analysis of their Potential Relevance to Abiotic Stress Tolerance and Secondary Metabolite Biosynthesis. Front Plant Sci. 2018;9:64.

51. Zhao F, Li G, Hu P, Zhao X, Li L, Wei W, et al. Identification of basic/helix-loop-helix transcription factors reveals candidate genes involved in anthocyanin biosynthesis from the strawberry whiteflesh mutant. Sci Rep. 2018;8(1):2721.

52. Feng XM, Zhao Q, Zhao LL, Qiao Y, Xie XB, Li HF, et al. The cold-induced basic helix-loop-helix transcription factor gene $\mathrm{MdClbHLH1}$ encodes an ICE-like protein in apple. BMC Plant Biology. 2012;12(1):22.

53. Zhao H, Wang X, Zhu D, Cui S, Li X, Cao Y, et al. A single amino acid substitution in Illf subfamily of basic helix-loop-helix transcription factor AtMYC1 leads to trichome and root hair patterning defects by abolishing its interaction with partner proteins in Arabidopsis. J Biol Chem. 2012;287(17):1410914121.

54. Hichri I, Heppel SC, Pillet J, Leon C, Czemmel S, Delrot S, et al. The basic helix-loop-helix transcription factor MYC1 is involved in the regulation of the flavonoid biosynthesis pathway in grapevine. Mol Plant. 2010;3(3):509-523.

55. Kiribuchi K, Jikumaru Y, Kaku H, Minami E, Hasegawa M, Kodama O, et al. Yamane H. Involvement of the basic helix-loop-helix transcription factor RERJ1 in wounding and drought stress responses in rice plants. Biosci Biotechnol Biochem. 2005;69:1042-1044.

56. Jiang Y, Yang B, Deyholos MK. Functional characterization of the Arabidopsis bHLH92 transcription factor in abiotic stress. Mol Genet Genomics. 2009;282(5):503-516.

57. Chen L, Chen Y, Jiang J, Chen S, Chen F, Guan Z, Fang W. The constitutive expression of Chrysanthemum dichrum ICE1 in Chrysanthemum grandiflorum improves the level of low temperature, salinity and drought tolerance. Plant Cell Rep. 2012;31(9):1747-1758.

58. An JP, Zhang XW, Bi SQ, You CX, Wang XF, Hao YJ. MdbHLH93, an apple activator regulating leaf senescence, is regulated by ABA and MdBT2 in antagonistic ways. New Phytol. 2019:222(2):735751.

59. Crooks GE, Hon G, Chandonia JM, Brenner SE. WebLogo: a sequence logo generator. Genome Res. 2004;14:1188-1190.

60. Kumar S, Stecher G, Tamura K. MEGA7: Molecular Evolutionary Genetics Analysis Version 7.0 for Bigger Datasets. Mol Biol Evol. 2016;33(7):1870-1874. 
61. Guo AY, Zhu QH, Chen X, Luo JC. GSDS: A gene structure display server. Yi Chuan. 2007;29:10231026.

62. Wang Y, Tang H, DeBarry JD, Tan X, Li J, Wang X, et al. MCScanX: A toolkit for detection and evolutionary analysis of gene synteny and collinearity. Nucleic Acids Res. 2012;40:e49.

63. Lescot M, Dehais P, Thijs G, Marchal K, Moreau Y, De Peer YV, et al. PlantCARE, a database of plant cis-acting regulatory elements and a portal to tools for in silico analysis of promoter sequences. Nucleic Acids Res. 2002;30:325-327.

64. Livak KJ, Schmittgen TD. Analysis of relative gene expression data using Real-Time Quantitative PCR and the 2. $\Delta \Delta$ Ct method.Methods. 2001;25:402-408.

65. Liu M, Zhang C, Duan L, Luan Q, Li J, Yang A, et al. CsMYB60 is a key regulator of flavonols and proanthocyanidans that determine the colour of fruit spines in cucumber. J Exp Bot. 2019;70(1):6984.

\section{Table}

\section{Table $1 b H L H$ genes in Cucumber}




\begin{tabular}{|c|c|c|c|c|c|c|}
\hline CsbHLH & Gene ID & Gene length & \multicolumn{3}{|c|}{ Amino acid length } & $\mathrm{pI}$ \\
\hline 001 & CsaV3_1G005290 & Chr1:3503806-3504411 & 1909 & 84 & 5.04 & \\
\hline 002 & CsaV3_1G011300 & Chr1:6972358-6976069 & 4167 & 236 & 5.78 & \\
\hline 003 & CsaV3_3G022420 & Chr3:19737551-19739874 & 958 & 502 & 5.7 & \\
\hline 004 & CsaV3_3G049150 & Chr3:40071324-40074834 & 1400 & 689 & 5.11 & \\
\hline 005 & CsaV3_3G001710 & Chr3:1295970-1297898 & 5601 & 643 & 6.21 & \\
\hline 006 & CsaV3_3G000850 & Chr3:656062-658628 & 6057 & 448 & 8.65 & \\
\hline 007 & CsaV3_1G039580 & Chr1:24945063-24953384 & 1684 & 319 & 5.78 & \\
\hline 008 & CsaV3_2G007370 & Chr2:3725743-3731272 & 605 & 707 & 6.09 & \\
\hline 009 & CsaV3_4G032110 & Chr4:22635255-22641052 & 3486 & 551 & 6.25 & \\
\hline 010 & CsaV3_1G043790 & Chr1:28804330-28811620 & 6167 & 960 & 6.4 & \\
\hline 011 & CsaV3_2G015700 & Chr2:13017831-13023291 & 1802 & 336 & 5.64 & \\
\hline 012 & CsaV3_6G000530 & Chr6:351129-356108 & 2050 & 645 & 5.51 & \\
\hline 013 & CsaV3_3G007980 & Chr3:6919411-6921670 & 2306 & 650 & 5.83 & \\
\hline 014 & CsaV3_2G010120 & Chr2:6877325-6878870 & 2524 & 323 & 6.02 & \\
\hline 015 & CsaV3_7G025510 & Chr7:14980031-14984292 & 3589 & 533 & 6.06 & \\
\hline 016 & CsaV3_6G009090 & Chr6:7311297-7315687 & 3711 & 486 & 6.35 & \\
\hline 017 & CsaV3_2G028950 & Chr2:18953613-18956324 & 3528 & 348 & 8.3 & \\
\hline 018 & CsaV3_7G007460 & Chr7:4647975-4650800 & 3316 & 335 & 6.64 & \\
\hline 019 & CsaV3_6G044570 & Chr6:26373287-26375739 & 3723 & 330 & 5.3 & \\
\hline 020 & CsaV3_6G044560 & Chr6:26366139-26368813 & 11538 & 309 & 5.81 & \\
\hline 021 & CsaV3_5G026500 & Chr5:21650877-21655135 & 2678 & 624 & 5.04 & \\
\hline 022 & CsaV3_6G044730 & Chr6:26485325-26487050 & 1415 & 342 & 4.86 & \\
\hline 023 & CsaV3_2G030090 & Chr2:19685080-19689447 & 4059 & 363 & 4.57 & \\
\hline 024 & CsaV3_6G043370 & Chr6:25541506-25544586 & 8321 & 416 & 5.2 & \\
\hline 025 & CsaV3_6G044580 & Chr6:26382822-26384588 & 3478 & 276 & 6.05 & \\
\hline 026 & CsaV3_2G035250 & Chr2:23586273-23591605 & 3855 & 239 & 6.77 & \\
\hline 027 & CsaV3_2G008770 & Chr2:5179234-5186154 & 7290 & 246 & 4.92 & \\
\hline 028 & CsaV3_6G008940 & Chr6:7177946-7179613 & 5695 & 432 & 5.42 & \\
\hline 029 & CsaV3_6G014370 & Chr6:10430376-10432021 & 6113 & 308 & 4.98 & \\
\hline 030 & CsaV3_5G033960 & Chr5:27092009-27094880 & 4184 & 372 & 5.77 & \\
\hline 031 & CsaV3_6G036080 & Chr6:20032486-20036831 & 1493 & 242 & 9.03 & \\
\hline 032 & CsaV3_1G033410 & Chr1:20481133-20483811 & 5529 & 256 & 9.24 & \\
\hline 033 & CsaV3_1G009900 & Chr1:6174783-6178372 & 6920 & 551 & 5.6 & \\
\hline 034 & CsaV3_2G001440 & Chr2:370393-376506 & 1545 & 543 & 8.37 & \\
\hline 035 & CsaV3_1G031920 & Chr1:18957508-18969046 & 2275 & 242 & 5.03 & \\
\hline 036 & CsaV3_7G004510 & Chr7:3234760-3236331 & 1320 & 211 & 6.77 & \\
\hline 037 & CsaV3_4G034440 & Chr4:24394485-24395550 & 3489 & 239 & 7.1 & \\
\hline 038 & CsaV3_4G029740 & Chr4:19342473-19344831 & 5460 & 253 & 6.17 & \\
\hline 039 & CsaV3_3G000950 & Chr3:733695-738681 & 1011 & 773 & 5.11 & \\
\hline 040 & CsaV3_2G026610 & Chr2:18201502-18202748 & 2627 & 240 & 7.26 & \\
\hline 041 & CsaV3_1G040580 & Chr1:25826012-25829490 & 1816 & 492 & 7.03 & \\
\hline 042 & CsaV3_6G037080 & Chr6:20849605-20855801 & 4137 & 651 & 5.91 & \\
\hline 043 & CsaV3_6G041730 & Chr6:24304724-24306996 & 1246 & 235 & 6.42 & \\
\hline 044 & CsaV3_1G003910 & Chr1:2423148-2424832 & 2711 & 266 & 6.87 & \\
\hline 045 & CsaV3_3G013690 & Chr3:10293079-10294740 & 2245 & 191 & 8.64 & \\
\hline 046 & CsaV3_1G006280 & Chr1:4002735-4008902 & 4367 & 566 & 9.01 & \\
\hline 047 & CsaV3_1G002260 & Chr1:1450554-1451954 & 1411 & 261 & 6.16 & \\
\hline 048 & CsaV3_3G039100 & Chr3:32125102-32130846 & 2115 & 370 & 5.74 & \\
\hline 049 & CsaV3_5G033600 & Chr5:26846039-26850063 & 5332 & 571 & 6.09 & \\
\hline 050 & CsaV3_1G006650 & Chr1:4277323-4279125 & 2566 & 279 & 6.1 & \\
\hline
\end{tabular}




\begin{tabular}{|c|c|c|c|c|c|}
\hline 051 & CsaV3_6G001900 & Chr6:1303975-1307218 & 4986 & 248 & 9.26 \\
\hline 052 & CsaV3_1G037610 & Chr1:23567571-23568986 & 1928 & 309 & 4.78 \\
\hline 053 & CsaV3_6G037070 & Chr6:20836169-20845986 & 5647 & 263 & 6.08 \\
\hline 054 & CsaV3_2G026190 & Chr2:17969523-17971339 & 1844 & 330 & 5.15 \\
\hline 055 & CsaV3_3G034600 & Chr3:29292016-29296383 & 2259 & 695 & 5.24 \\
\hline 056 & CsaV3_3G044120 & Chr3:35995420-35997451 & 4852 & 272 & 5.09 \\
\hline 057 & CsaV3_2G005070 & Chr2:2751170-2752663 & 3663 & 318 & 5.74 \\
\hline 058 & CsaV3_2G014750 & Chr2:12321166-12324655 & 1661 & 343 & 6.2 \\
\hline 059 & CsaV3_7G027630 & Chr7:17195587-17199883 & 1509 & 317 & 6 \\
\hline 060 & CsaV3_2G025890 & Chr2:17778099-17780726 & 3295 & 342 & 6.26 \\
\hline 061 & CsaV3_2G030500 & Chr2:20027274-20029389 & 2181 & 372 & 4.71 \\
\hline 062 & CsaV3_3G015900 & Chr3:11810548-11813843 & 2822 & 547 & 6.88 \\
\hline 063 & CsaV3_7G000080 & Chr7:185656-189146 & 2627 & 457 & 5.85 \\
\hline 064 & CsaV3_1G000190 & Chr1:132006-133915 & 2323 & 276 & 7.03 \\
\hline 065 & CsaV3_3G028610 & Chr3:25161463-25169489 & 3059 & 540 & 5.7 \\
\hline 066 & CsaV3_2G003660 & Chr2:1833037-1837221 & 6474 & 422 & 6.1 \\
\hline 067 & CsaV3_4G002800 & Chr4:1745825-1749669 & 8026 & 168 & 7.65 \\
\hline 068 & CsaV3_1G045830 & Chr1:31650489-31656184 & 4367 & 395 & 6.39 \\
\hline 069 & CsaV3_4G026430 & Chr4:15704947-15706451 & 3065 & 196 & 6.51 \\
\hline 070 & CsaV3_3G007090 & Chr3:6381666-6383510 & 5744 & 359 & 5.94 \\
\hline 071 & CsaV3_3G049050 & Chr3:40003473-40010806 & 1802 & 322 & 7.73 \\
\hline 072 & CsaV3_1G005810 & Chr1:3727778-3731264 & 2031 & 443 & 9.02 \\
\hline 073 & CsaV3_2G026540 & Chr2:18154564-18158701 & 3855 & 380 & 5.01 \\
\hline 074 & CsaV3_4G035310 & Chr4:24884098-24888333 & 2900 & 403 & 5.7 \\
\hline 075 & CsaV3_3G020750 & Chr3:16961726-16964548 & 7333 & 248 & 7.09 \\
\hline 076 & CsaV3_5G018750 & Chr5:14293739-14297926 & 3510 & 534 & 5.2 \\
\hline 077 & CsaV3_4G034660 & Chr4:24537693-24539435 & 1700 & 409 & 6.38 \\
\hline 078 & CsaV3_7G026520 & Chr7:16047841-16051016 & 3844 & 490 & 6.04 \\
\hline 079 & CsaV3_1G001960 & Chr1:1286661-1290828 & 5186 & 196 & 7.64 \\
\hline 080 & CsaV3_5G040480 & Chr5:31836009-31840665 & 2228 & 245 & 5.67 \\
\hline 081 & CsaV3_6G002130 & Chr6:1472037-1473566 & 1504 & 161 & 5.12 \\
\hline 082 & CsaV3_1G028780 & Chr1:15708212-15711935 & 2358 & 423 & 6.34 \\
\hline 083 & CsaV3_5G024030 & Chr5:18711066-18713891 & 3741 & 285 & 6.21 \\
\hline 084 & CsaV3_4G000380 & Chr4:228607-230307 & 5797 & 342 & 4.89 \\
\hline 085 & CsaV3_4G034980 & Chr4:24696914-24698535 & 1065 & 245 & 6.18 \\
\hline 086 & CsaV3_3G014190 & Chr3:10642450-10643959 & 1742 & 203 & 7.81 \\
\hline 087 & CsaV3_7G035000 & Chr7:22142359-22144409 & 1621 & 394 & 6.54 \\
\hline 088 & CsaV3_2G016810 & Chr2:14091802-14092813 & 4235 & 216 & 9.77 \\
\hline 089 & CsaV3_6G012850 & Chr6:8973920-8976038 & 2826 & 262 & 9.23 \\
\hline 090 & CsaV3_5G031540 & Chr5:25725825-25732273 & 1729 & 679 & 7.3 \\
\hline 091 & CsaV3_2G011050 & Chr2:8296805-8299080 & 1004 & 496 & 5.5 \\
\hline 092 & CsaV3_2G013060 & Chr2:10626443-10627763 & 5030 & 280 & 9.14 \\
\hline 093 & CsaV3_3G048260 & Chr3:39394424-39397324 & 4187 & 326 & 4.94 \\
\hline 094 & CsaV3_1G039160 & Chr1:24662874-24666933 & 2825 & 359 & 5.94 \\
\hline 095 & CsaV3_7G008580 & Chr7:5322491-5324405 & 2588 & 254 & 7.22 \\
\hline 096 & CsaV3_2G029940 & Chr2:19575197-19577442 & 4258 & 308 & 6.01 \\
\hline 097 & CsaV3_3G011010 & Chr3:8707121-8711973 & 6448 & 382 & 5.08 \\
\hline 098 & CsaV3_3G021970 & Chr3:19073471-19076098 & 2460 & 377 & 5.08 \\
\hline 099 & CsaV3_6G028530 & Chr6:16812784-16815830 & 2030 & 207 & 11.79 \\
\hline 100 & CsaV3_6G037460 & Chr6:21181685-21184516 & 4024 & 299 & 5.66 \\
\hline 101 & CsaV3_4G029750 & Chr4:19361035-19364776 & 2871 & 211 & 9.21 \\
\hline
\end{tabular}




\begin{tabular}{|c|c|c|c|c|c|}
\hline 102 & CsaV3_6G033930 & Chr6:18737868-18742274 & 1923 & 333 & 5.71 \\
\hline 103 & CsaV3_6G036240 & Chr6:20141242-20142683 & 4656 & 97 & 9.18 \\
\hline 104 & CsaV3_3G012210 & Chr3:9421374-9425037 & 4979 & 227 & 5.7 \\
\hline 105 & CsaV3_3G022870 & Chr3:20405212-20408271 & 3243 & 236 & 6.13 \\
\hline 106 & CsaV3_3G042970 & Chr3:34884792-34886594 & 1529 & 244 & 8.4 \\
\hline 107 & CsaV3_6G018830 & Chr6:13512926-13515085 & 1667 & 253 & 7.06 \\
\hline 108 & CsaV3_2G030310 & Chr2:19895721-19897132 & 4390 & 240 & 9.2 \\
\hline 109 & CsaV3_1G002670 & Chr1:1668618-1674219 & 2118 & 359 & 8.65 \\
\hline 110 & CsaV3_3G045440 & Chr3:37108680-37112535 & 1645 & 430 & 6.69 \\
\hline 111 & CsaV3_5G012430 & Chr5:7900420-7905450 & 2159 & 451 & 6.33 \\
\hline 112 & CsaV3_1G011460 & Chr1:7106592-7110120 & 3046 & 360 & 4.75 \\
\hline 113 & CsaV3_7G008090 & Chr7:5057906-5059973 & 4406 & 255 & 6.11 \\
\hline 114 & CsaV3_5G026380 & Chr5:21538651-21541239 & 4345 & 173 & 9.15 \\
\hline 115 & CsaV3_UNG229040 & scaffold115:93241-95547 & 1441 & 274 & 9.42 \\
\hline 116 & CsaV3_3G027730 & Chr3:24067204-24073678 & 9817 & 529 & 5.88 \\
\hline 117 & CsaV3_7G003870 & Chr7:2853486-2854424 & 6196 & 313 & 5.32 \\
\hline 118 & CsaV3_3G016560 & Chr3:12365460-12367641 & 2831 & 253 & 8.6 \\
\hline 119 & CsaV3_1G012350 & Chr1:7668571-7671887 & 2272 & 205 & 6.16 \\
\hline 120 & CsaV3_5G003430 & Chr5:2204438-2205442 & 3080 & 256 & 7.75 \\
\hline 121 & CsaV3_6G047120 & Chr6:27815147-27818761 & 2674 & 337 & 6.13 \\
\hline 122 & CsaV3_1G042640 & Chr1:27559193-27563048 & 2452 & 438 & 7.72 \\
\hline 123 & CsaV3_3G005540 & Chr3:4716554-4722201 & 1766 & 439 & 6.41 \\
\hline 124 & CsaV3_6G046660 & Chr6:27537459-27541954 & 1725 & 357 & 8.44 \\
\hline 125 & CsaV3_5G003420 & Chr5:2191536-2193265 & 1497 & 261 & 5.59 \\
\hline 126 & CsaV3_5G003410 & Chr5:2180337-2183163 & 4495 & 252 & 7.01 \\
\hline 127 & CsaV3_6G049510 & Chr6:28902722-28905982 & 3614 & 405 & 5.35 \\
\hline 128 & CsaV3_4G003860 & Chr4:2368080-2373266 & 3260 & 357 & 8.51 \\
\hline 129 & CsaV3_7G031270 & Chr7:19779388-19783470 & 749 & 420 & 8.28 \\
\hline 130 & CsaV3_3G039080 & Chr3:32107556-32110621 & 3490 & 367 & 9.16 \\
\hline 131 & CsaV3_6G045070 & Chr6:26672336-26673833 & 938 & 229 & 10.26 \\
\hline 132 & CsaV3_6G051560 & Chr6:29996501-29997250 & 1571 & 250 & 5.66 \\
\hline 133 & CsaV3_7G027460 & Chr7:17033739-17042484 & 2825 & 692 & 5.66 \\
\hline 134 & CsaV3_5G037950 & Chr5:30085680-30087603 & 1391 & 92 & 9.09 \\
\hline 135 & CsaV3_1G002240 & Chr1:1440343-1441301 & 2067 & 93 & 9.09 \\
\hline 136 & CsaV3_5G032530 & Chr5:26313766-26315796 & 1914 & 96 & 9.17 \\
\hline 137 & CsaV3_1G009880 & Chr1:6153304-6155828 & 4261 & 373 & 6.61 \\
\hline 138 & CsaV3_7G033460 & Chr7:21082838-21087117 & 3175 & 298 & 6.78 \\
\hline 139 & CsaV3_7G007860 & Chr7:4913547-4914938 & 8745 & 211 & 6.35 \\
\hline 140 & CsaV3_4G010010 & Chr4:7769516-7771744 & 4296 & 333 & 5.11 \\
\hline 141 & CsaV3_1G003270 & Chr1:2026829-2032886 & 4082 & 619 & 9.19 \\
\hline 142 & CsaV3_5G031750 & Chr5:25868912-25871372 & 4279 & 365 & 5.84 \\
\hline
\end{tabular}

\section{Figures}




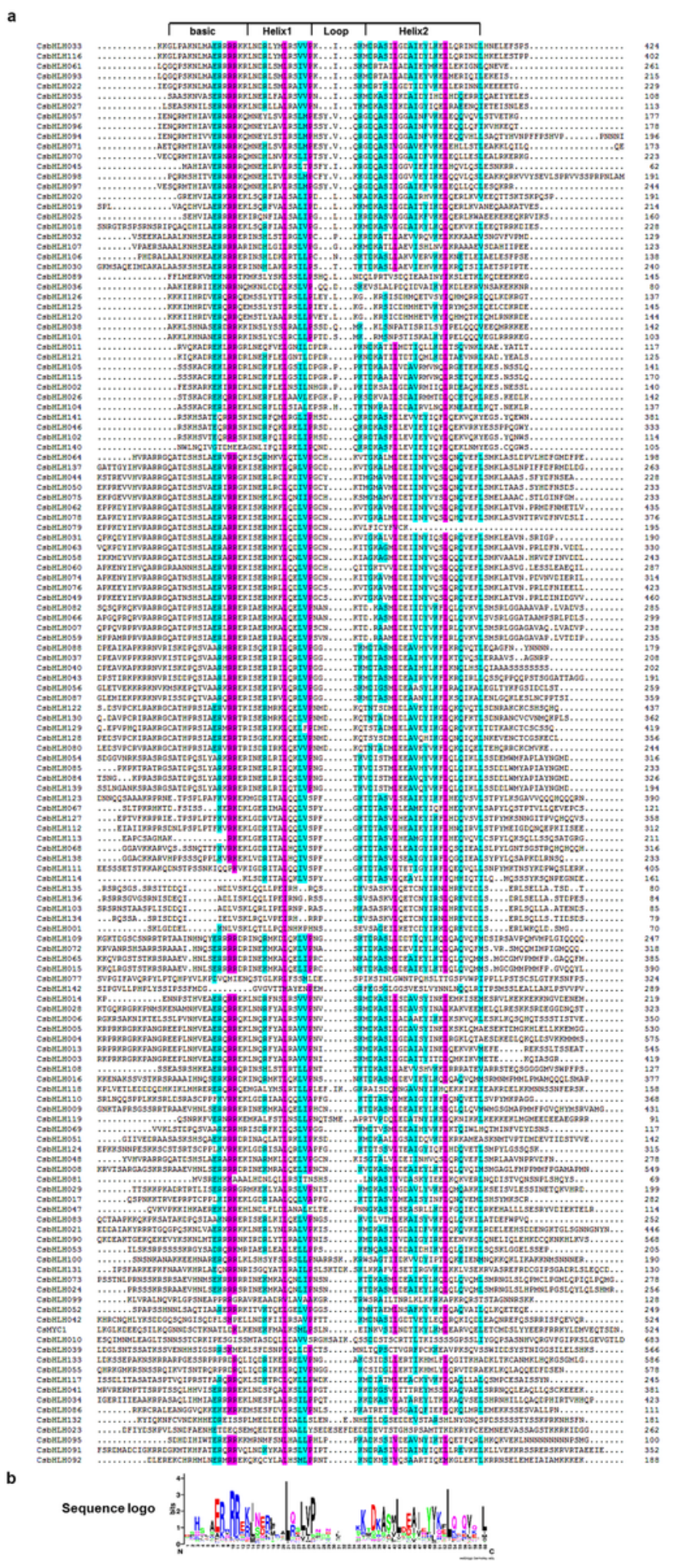

\section{Figure 1}

Conserved amino acids and multiple sequence alignment schematic diagrams of the CsbHLHs bHLH domains. (a) Multiple sequence alignments of $\mathrm{CsbHLH}$ proteins. The $\mathrm{CsbHLH}$ conserved sequences were marked with a purple background that amino acids identity was higher than $75 \%$, amino acids identity of more than $50 \%$ was marked with a light blue background. The bHLH domains were labeled. (b) Sequence 
logo of CsbHLH domains. The overall height of each stack represented the conservation of the sequence at that position.

a

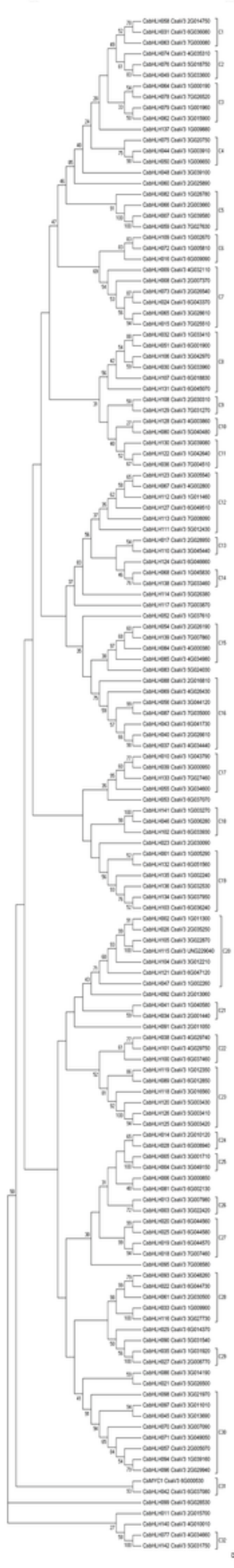

b
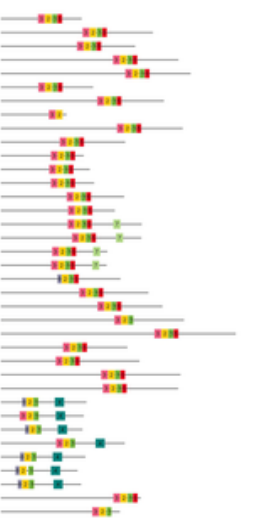

$c$

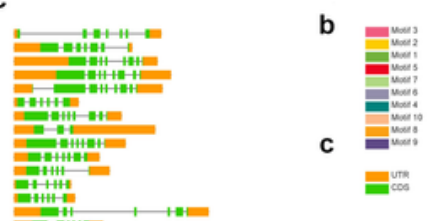

-
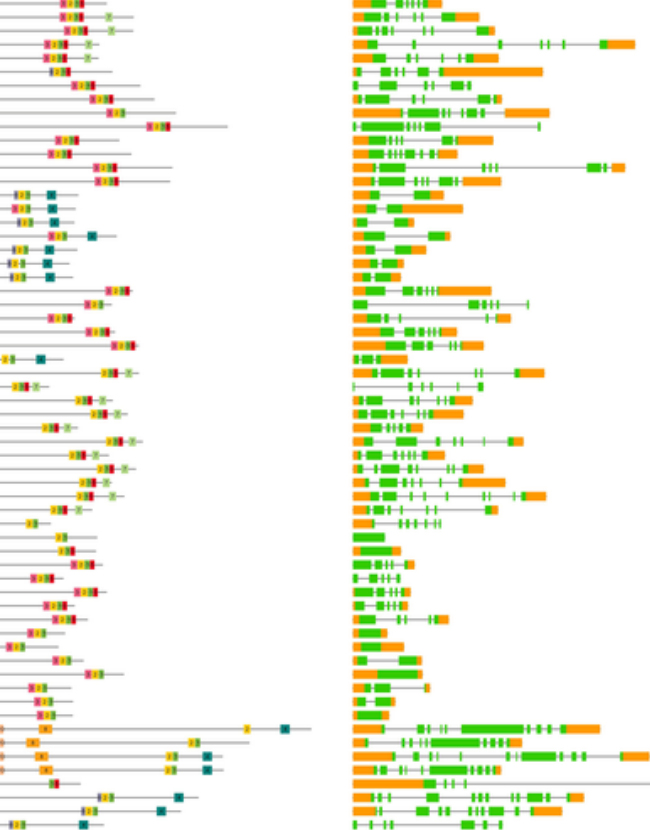

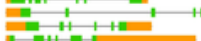

20

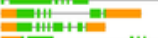

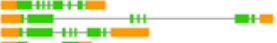

를

들

를

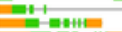

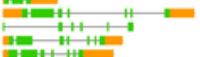

istin

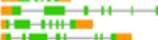

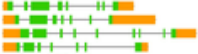

Ein+1

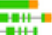

=

를

풀

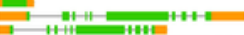

루

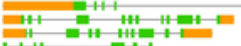

茪

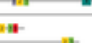

$\rightarrow$

ii:

$\sqrt{3}$

$\overbrace{10}^{m-19}$

$\min _{\rightarrow 11}^{\min }$

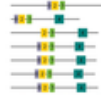
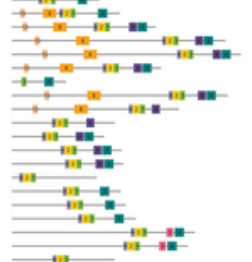

举

㱐:

,

橆!

早政

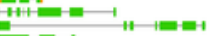

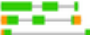

$=$
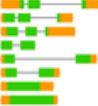

드름

달.

$=$

S.

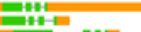

대:- 분

Fin:

"

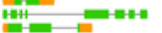

$=$

te-

E=-

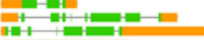

$1+\frac{1}{1+2}$

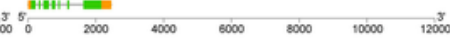

\section{Figure 2}

Phylogenetic relationships, gene structure and conserved protein motifs in bHLH genes from cucumber. (a) The phylogenetic tree was constructed based on the full-length protein sequences of $142 \mathrm{CsbHLH}$ proteins using MEGA 7.0 software. The tree showed the 32 phylogenetic subgroups (C1-C32) with high 
bootstrap value. (b) Conserved motifs in CsbHLH proteins. The motifs, numbers 1-10, were displayed in different colored boxes. The sequence logos and E values for each motif were given in Fig. S1. (c) Exonintron structure of $\mathrm{CsbHLH}$ genes. Exons and introns were indicated by green boxes and single lines, respectively. Blue boxes represented upstream or downstream. The length of each gene was listed in the Table 1.

a
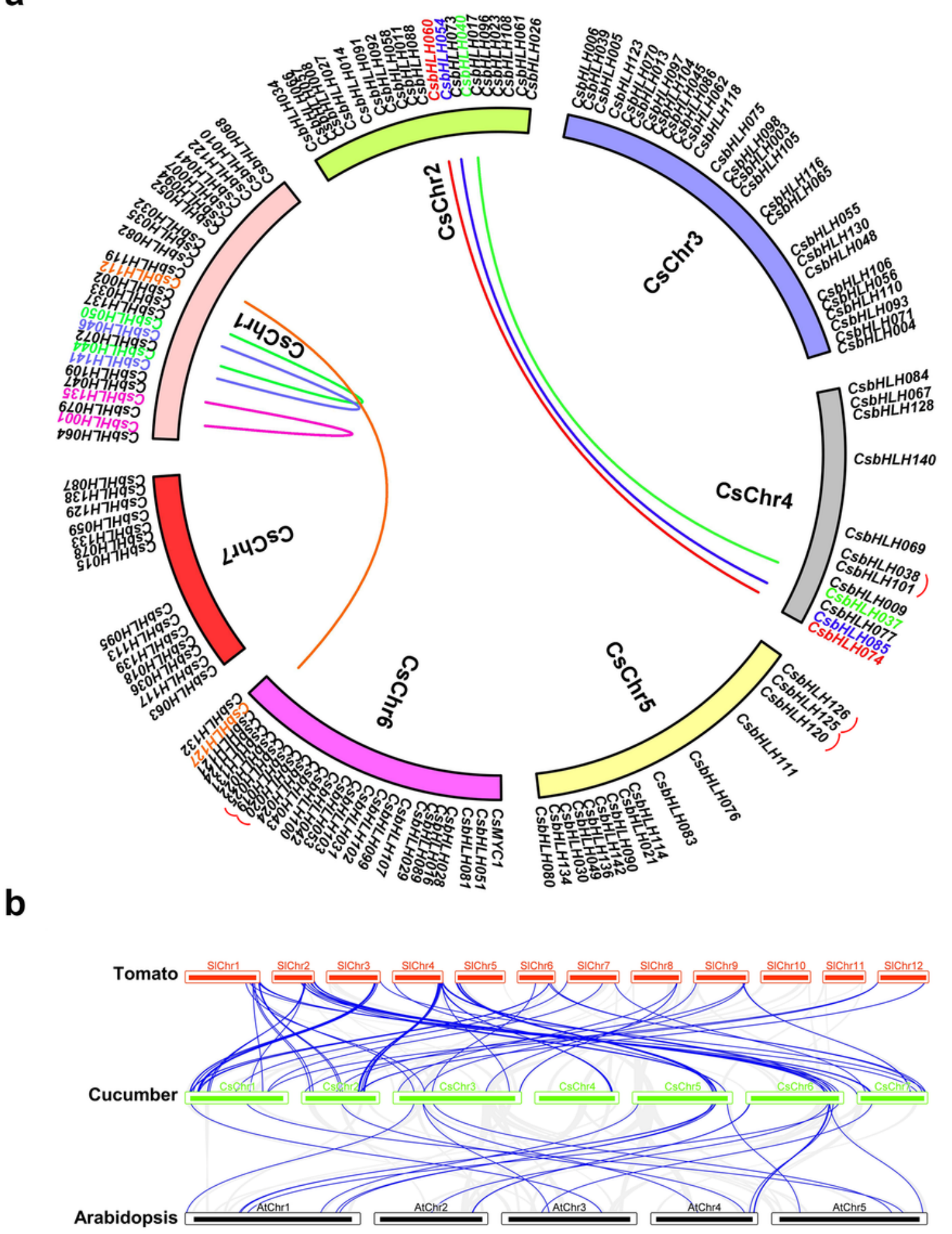

Figure 3 
Gene duplication and synteny analysis of CsbHLH genes. (a) Schematic representations for the chromosomal distribution and interchromosomal relationships of CsbHLH genes. Different line colors represented different segmental duplicated CsbHLH gene pairs, which the two genes of the same segmental duplicated gene pair were labeled in the same color. The red lines in the outer ring indicated tandem duplication gene pairs. (b) Synteny analysis of bHLH genes between cucumber and Arabidopsis and Tomato. Blue lines indicated the collinear blocks of bHLH gene within cucumber and Arabidopsis and Tomato genomes.

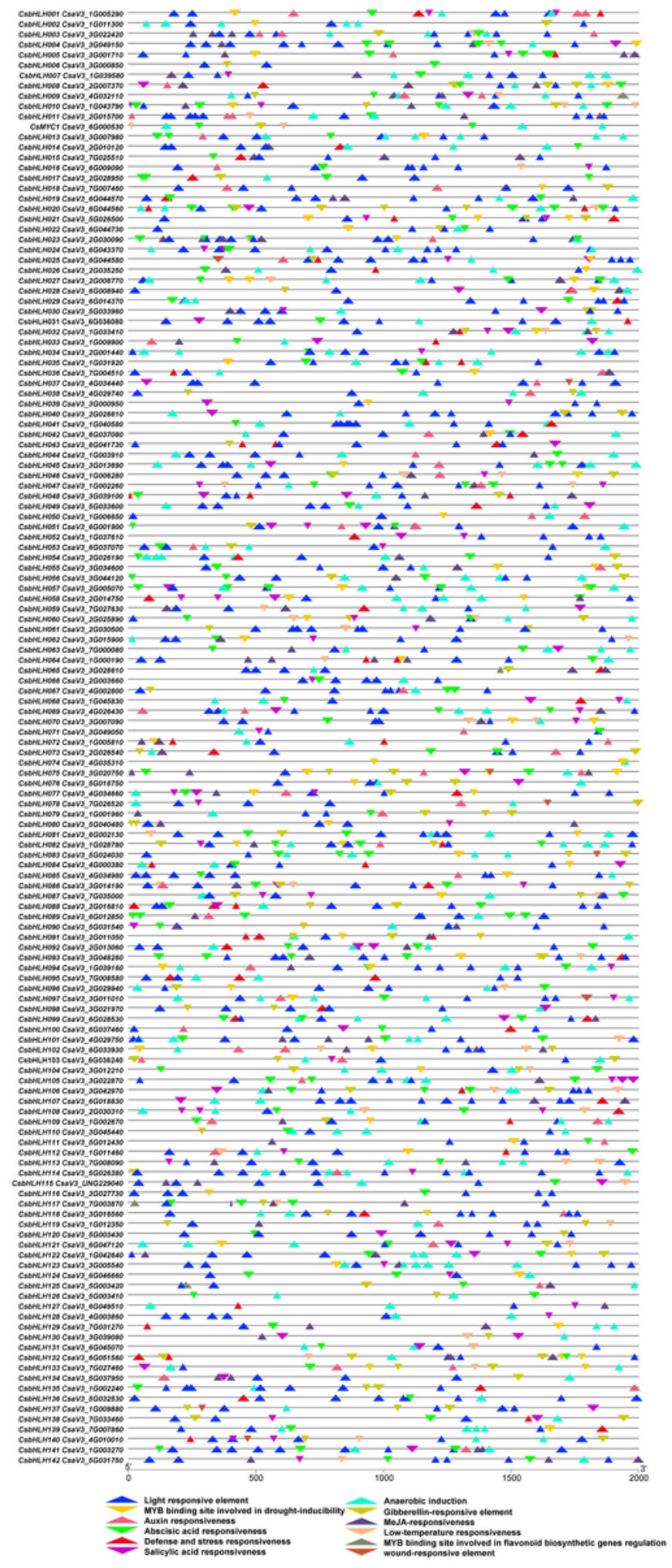




\section{Figure 4}

Cis-element analysis in the CsbHLH genes promoter regions. The potential cis-regulatory elements in the promoter regions 2-kb upstream of the CsbHLH genes, particularly the elements related to stress responsiveness (light induction; anaerobic induction, low temperature and drought inducibility) and plant hormones (Abscisic acid, Auxin, MeJA, Gibberellic acid and Salicylic acid). Different colored triangles represented different cis-elements.

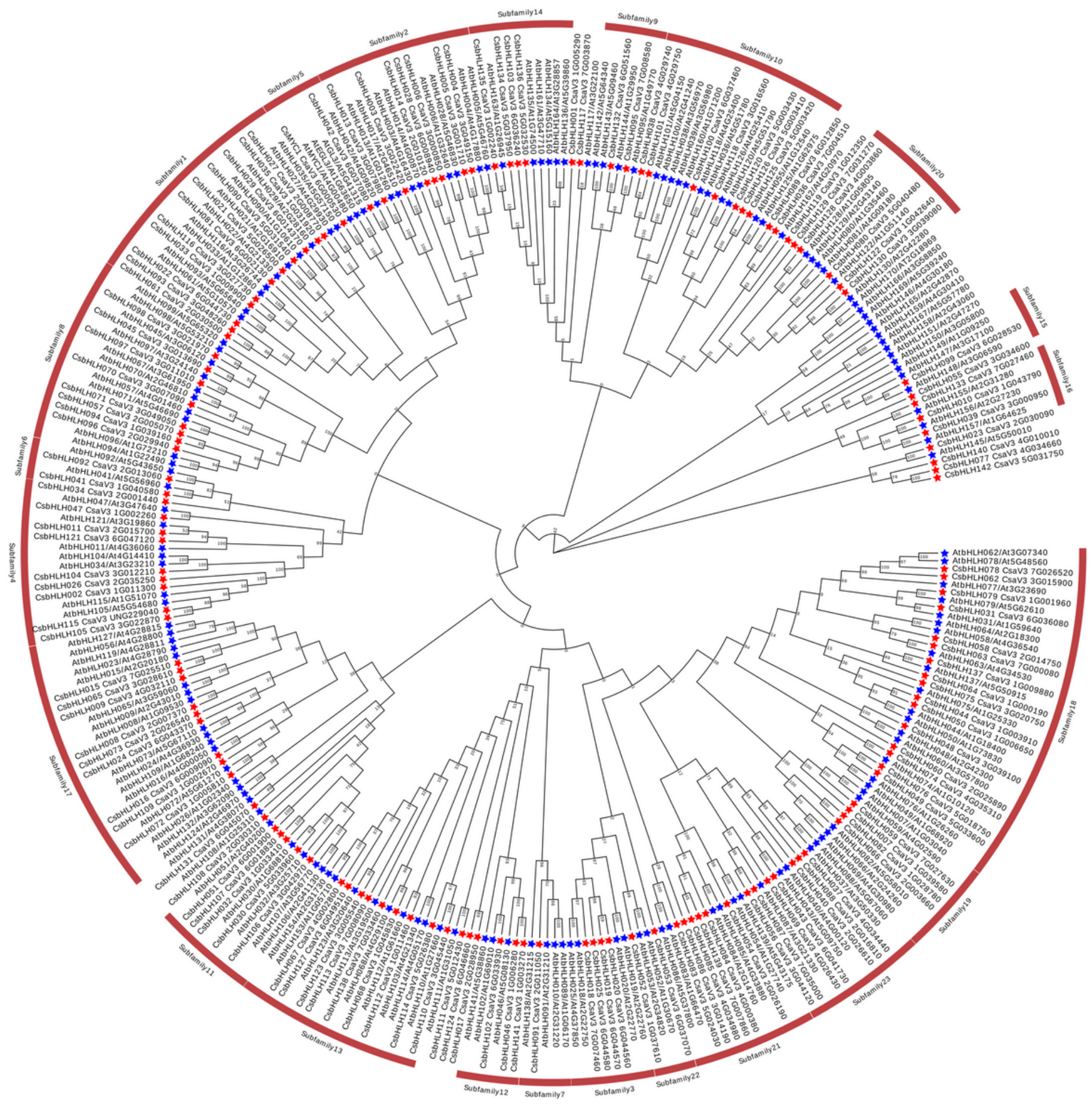

Figure 5 
Evolutionary tree analysis (circle tree) and subfamily classifications of bHLHs proteins in cucumber and Arabidopsis thaliana. The evolutionary tree was constructed using the Neighbor-Joining method with 1000 bootstrap replication. The evolutionary distances were computed using poisson correction. The analysis involved 142 cucumber bHLH protein sequences and 166 Arabidopsis thaliana bHLH protein. Red stars represented the CsbHLH proteins and blue represented the AtbHLH proteins.
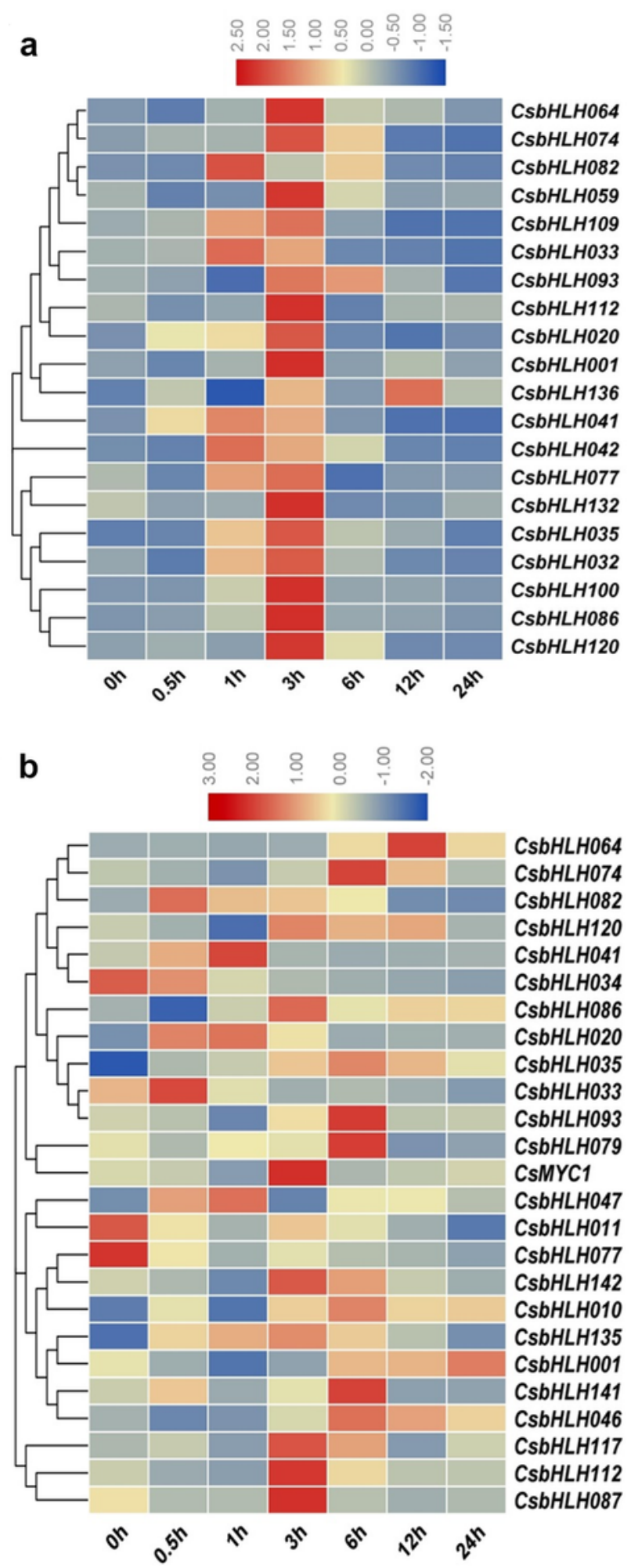

C

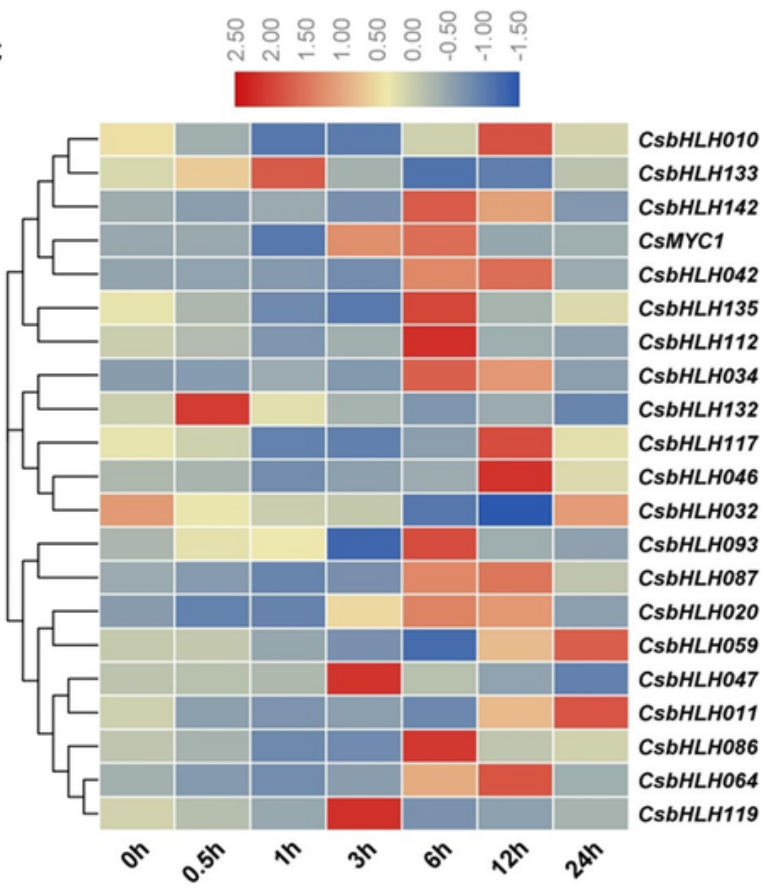

d
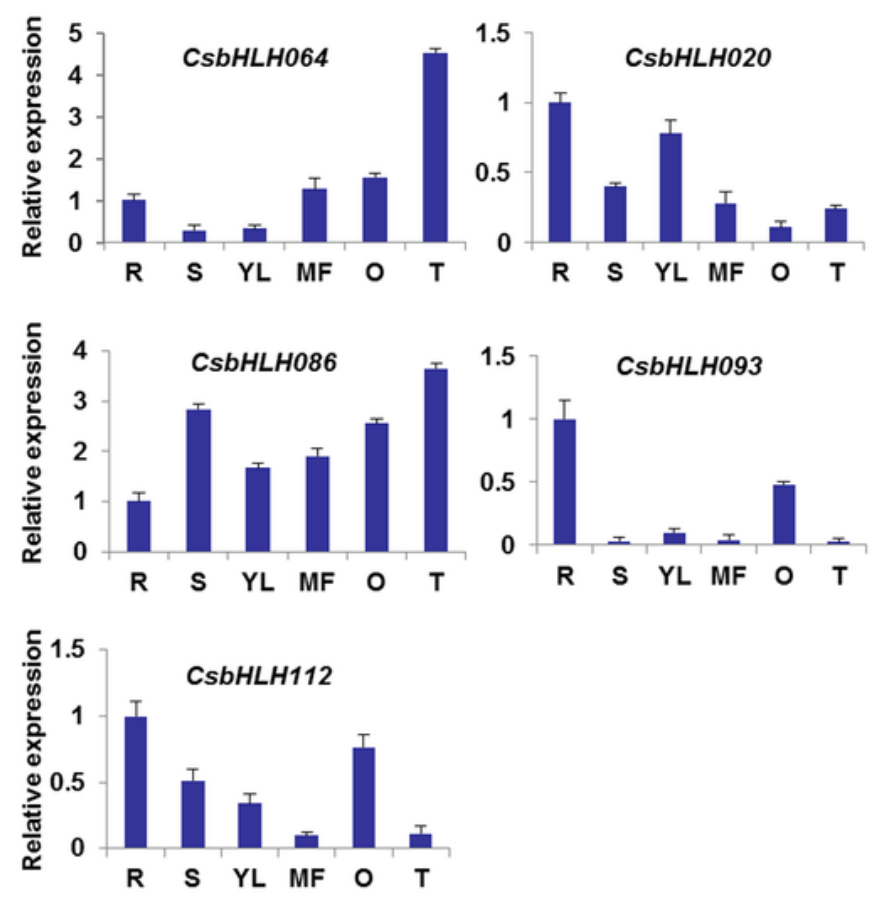

Figure 6 
Relative expression analysis of the CsbHLH genes under different stress conditions and different tissues. Expression patterns of $\mathrm{CsbHLH}$ genes under $\mathrm{NaCl}(100 \mathrm{mM})$ treatment (a), ABA $(100 \mu \mathrm{M})$ treatment (b) and low temperature $\left(4^{\circ} \mathrm{C}\right)$ treatment (c). (d) Tissue-specific expression profiles of five cucumber bHLH genes. Total RNA was isolated from roots $(R)$, stems $(S)$, young leaves $(Y L)$, male flowers $(M F)$, ovary $(0)$ and tendrils $(T)$, respectively. The cucumber $\beta$-actin gene was performed as an internal control, and three independent samples were used for these experiments. Error bars indicated standard errors (SE).

\section{Figure 7}

Response to salt and ABA stress between the overexpressed 35S empty vector and 35S:CsbHLH041 in cucumber seedings. (a) Salt stress tolerance comparison. Photographs of the $35 \mathrm{~S}$ empty vector and 35S:CsbHLH041 in cucumber seedings treated with $100 \mathrm{mM} \mathrm{NaCl}$ stress at different time periods during hydroponics. (b) ABA stress tolerance comparison. Photographs of the 35S and 35S:CsbHLH041 in cucumber seedings treated with $100 \mu \mathrm{M}$ ABA stress at different time periods during hydroponics.

\section{Figure 8}

CsbHLH041 transgenic Arabidopsis enhanced salt and ABA tolerance. (a) Relative expression of CsbHLH041 in Col-0 (WT) and two T3 generation transgenic lines by semi-quantitative PCR. The actin8 gene was used as an internal control. (b) Germination of WT seeds of Col-0 and CsbHLH041 transgenic lines $0 X-1,0 X-2$ on $1 / 2 \mathrm{MS}$ supplemented with $100 \mathrm{mM} \mathrm{NaCl}$ and $2 \mu \mathrm{M} \mathrm{ABA}$ after 7 days of cultivation at $22^{\circ} \mathrm{C}$. (c) and (d) Seed germination rate counted for corresponding (b), respectively. Three biological replications were performed. The bars showed the SD. Asterisks indicated a significant difference $* * \mathrm{p}<$ 0.01 compared with the corresponding controls. The growing of Col-0 (WT) and CsbHLH041 transgenic lines after $200 \mathrm{mM} \mathrm{NaCl}(\mathrm{e})$ and $100 \mu \mathrm{M} \mathrm{ABA}(\mathrm{f})$ treatments. 


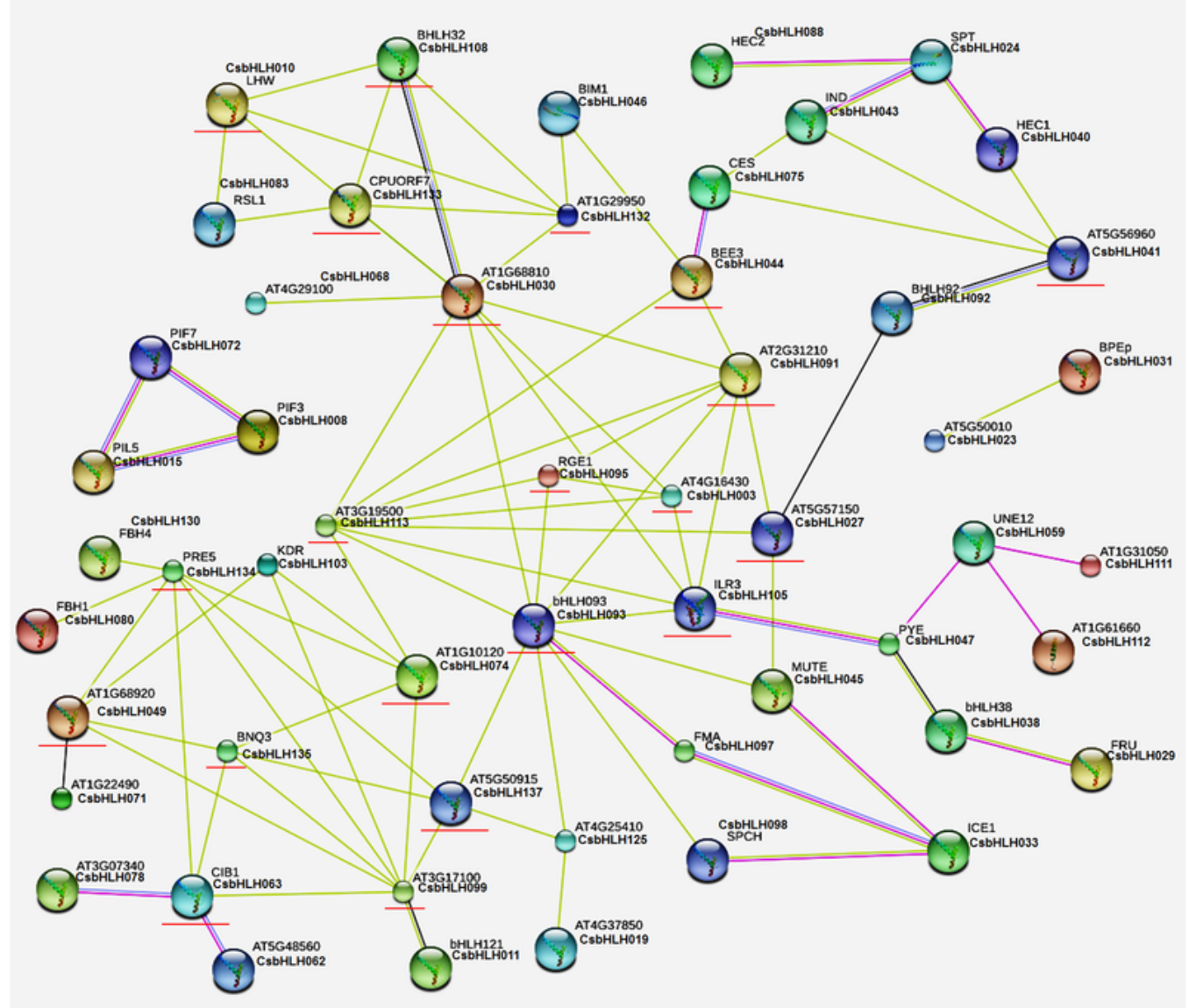

b
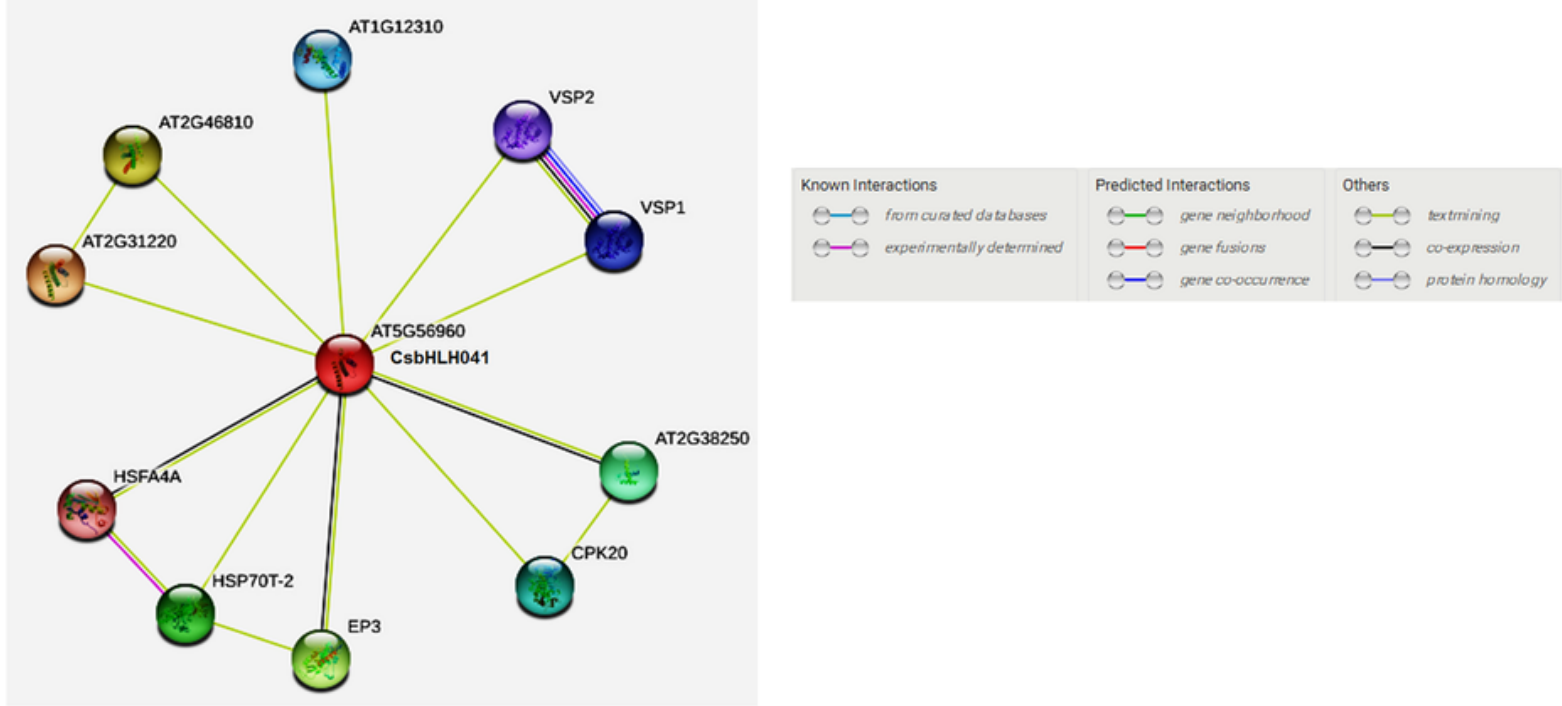

\section{Figure 9}

Protein interaction network for CsbHLHs based on CsbHLH orthologs in Arabidopsis. Protein interaction network predictions of CsbHLHs (a), CsbHLH041 (b), based on CsbHLH orthologs in Arabidopsis. Red lines indicated proteins that were predicted to interact with more than four other bHLH proteins. CsbHLH proteins were shown next to Arabidopsis orthologs. 


\section{Supplementary Files}

This is a list of supplementary files associated with this preprint. Click to download.

- TableS3.xlsx

- TableS6.xIsx

- TableS2.xlsx

- Additionalfile1.pdf

- TableS4.xIsx

- TableS7.xlsx

- TableS1.xIsx

- TableS5.xlsx 